\title{
Using DNA strand displacement to control interactions in DNA-grafted colloids
}

\section{Citation}

Gehrels, Emily W., William Rogers, and Vinothan N. Manoharan. 2018. "Using DNA Strand Displacement to Control Interactions in DNA-Grafted Colloids." Soft Matter. doi:10.1039/ c7sm01722g.

\section{Published Version}

doi:10.1039/C7SM01722G

\section{Permanent link}

http://nrs.harvard.edu/urn-3:HUL.InstRepos:34721961

\section{Terms of Use}

This article was downloaded from Harvard University's DASH repository, and is made available under the terms and conditions applicable to Open Access Policy Articles, as set forth at http:// nrs.harvard.edu/urn-3:HUL.InstRepos:dash.current.terms-of-use\#OAP

\section{Share Your Story}

The Harvard community has made this article openly available.

Please share how this access benefits you. Submit a story.

\section{Accessibility}




\section{Journal Name}

\section{ARTICLE TYPE}

Cite this: DOl: $10.1039 / x x x x x x x x x x$

\section{Using DNA strand displacement to control interac- tions in DNA-grafted colloids}

\author{
Emily W. Gehrels, ${ }^{a}$ W. Benjamin Rogers, ${ }^{a, b}$ and Vinothan N. Manoharan ${ }^{* a, c}$
}

Received Date

Accepted Date

DOI: 10.1039/xxxxxxxxxx

www.rsc.org/journalname

\begin{abstract}
Grafting DNA oligonucleotides to colloidal particles leads to specific, reversible interactions between those particles. However, the interaction strength varies steeply and monotonically with temperature, hindering the use of DNA-mediated interactions in self-assembly. We show how the dependence on temperature can be modified in a controlled way by incorporating DNA stranddisplacement reactions. The method allows us to make multicomponent systems that can selfassemble over a wide range of temperatures, invert the dependence on temperature to design colloidal systems that melt upon cooling, controllably transition between structures with different compositions, or design systems with multiple melting transitions. This wide range of behaviors can be realized simply by adding a small number of DNA strands to the solution, making the approach modular and straightforward to implement. We conclude with practical considerations for designing systems of DNA-mediated colloidal interactions.
\end{abstract}

\section{Introduction}

In this article we demonstrate how the interactions and phase behavior of DNA-grafted colloidal particles can be altered by adding free DNA strands that can hybridize with the grafted strands. We originally demonstrated this method in a short report in $2015 .{ }^{1}$ The present article is a more comprehensive study that extends on our previous work, offers new experimental results, describes the theory behind them, and discusses some practical considerations for using the method.

The underlying premise is that the usefulness of DNA-mediated colloidal interactions stems from their specificity and reversibility. ${ }^{2-4}$ Two particles grafted with DNA oligonucleotides bind ifand, in the absence of non-specific interactions, only if - the sequences are complementary, and the temperature is in a range where duplexes can form. The duplexes bridge the particles together, as shown in Figure 1a. Upon an increase in temperature, the bridges melt, and the particles dissociate. Lowering the temperature has the opposite effect. With this combination of specificity and reversibility, it is possible to design many singlecomponent and two-component systems that assemble into equilibrium crystals and clusters. ${ }^{4-14}$

However, these interactions are limited in one significant way: the binding strength decreases steeply and monotonically with in-

\footnotetext{
${ }^{a}$ Harvard John A. Paulson School of Engineering and Applied Sciences, Harvard University, Cambridge, Massachusetts 02138, USA.

${ }^{b}$ Martin Fisher School of Physics, Brandeis University, Waltham, Massachusetts 02453, USA

${ }^{c}$ Department of Physics, Harvard University, Cambridge, Massachusetts 02138 USA

* corresponding author, email: vnm@seas.harvard.edu
}

creasing temperature. ${ }^{15,16}$ Over a window of $1-2{ }^{\circ} \mathrm{C}$, the binding strength typically decreases from many times the thermal energy $k T$ to negligible. As a result, the fraction of unbound particles (the "singlet fraction") in a suspension decreases rapidly with decreasing temperature-much more rapidly than the singlet fraction of DNA strands that are identical to the ones on the particles, but are free in solution (Figure 1b).

This sharp transition makes it difficult for systems of particles to equilibrate. Equilibration happens only at temperatures where the DNA binding energies are strong enough to cause the particles to attract, but not so strong that the DNA bridges between the particles have long lifetimes. ${ }^{4,16,17}$ In this temperature regime, the single-stranded DNA (ssDNA) molecules causing the attraction bind and unbind on timescales short compared to the particle motion, allowing the particles to explore different configurations without becoming trapped or falling apart. Because the binding strength varies so steeply with temperature, equilibration can occur only within a narrow window of temperatures. Thus, the steep dependence of the interactions on temperature limits the benefits of DNA-mediated interactions: while it is possible to design a system with a particular equilibrium structure, it is challenging to get it to form that structure.

The problem becomes more acute for systems with several species of particles-where "species" means a set of particles grafted with a particular combination of oligonucleotides. In such systems, one can use the nearest-neighbor model of DNA thermodynamics ${ }^{18}$ to predict how the melting temperatures should change with the DNA sequences. The model, in conjunction with additional theory that we describe later, allows one to design se- 


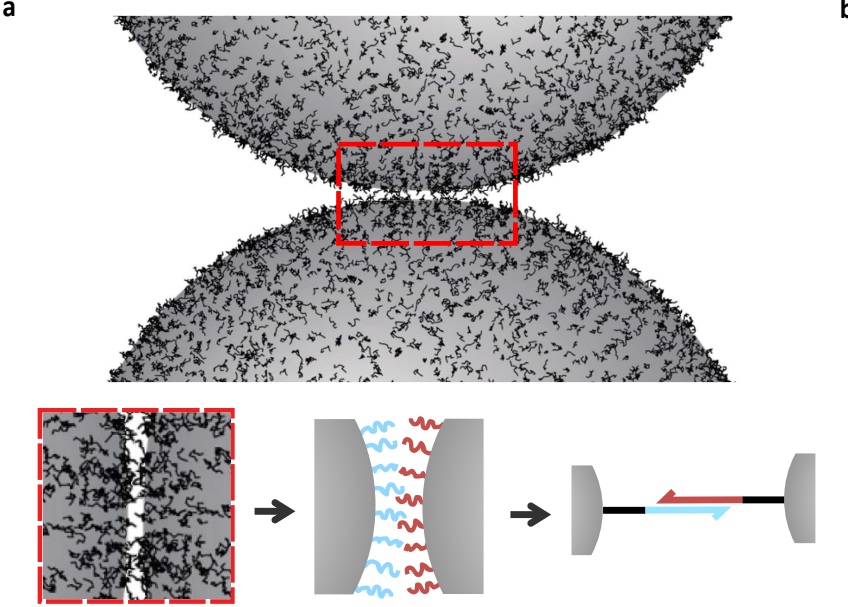

b

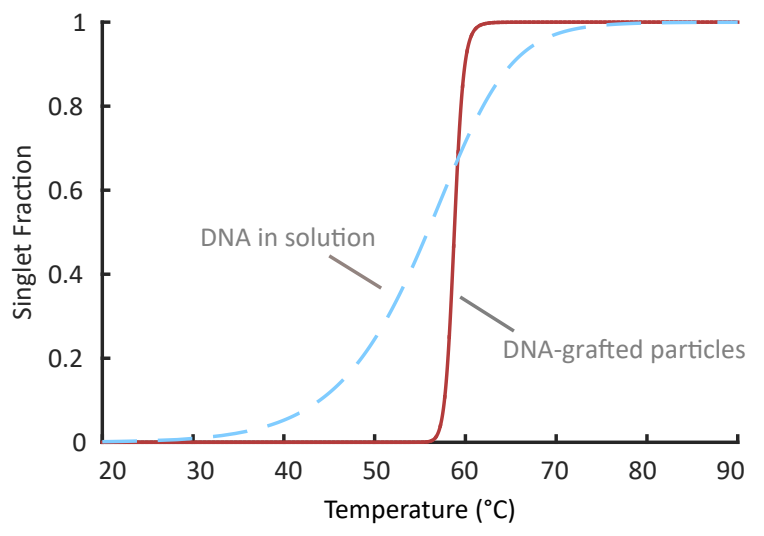

c

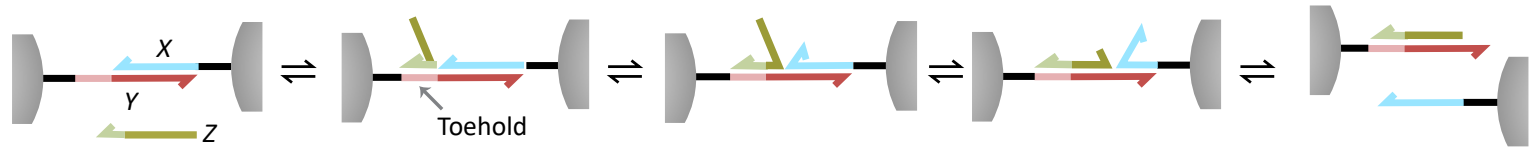

Fig. 1 a) A scale rendering, generated by Monte Carlo simulation of polymer chain configurations, of DNA-grafted 1- $\mu \mathrm{m}$ polystyrene particles. The length of the DNA strands is 65 bases, such that the radius of gyration $L$ is approximately $11 \mathrm{~nm}$. The bottom images show how we schematically depict the bridges that bind the particles together. In these schematics, a zoomed-in portion of each particle is shown in gray, and the strands are not drawn to scale. b) Theoretical singlet fraction as a function of temperature for DNA in solution (blue, dashed) and for particles with grafted strands (red, solid), calculated using Equations 2, 4, and 5. c) Schematic of toehold-mediated strand displacement. At left, two particles are bridged by complementary ssDNA $(X$ and $Y$ ). A displacing strand $(Z)$, which is also complementary to $Y$, can bind to an exposed region of $Y$ (the "toehold," shown in pink) and displace $X$ through a stochastic exchange of bases between the duplexes $X Y$ and $Y Z$. This process is reversible if there remains an exposed region of $Y$ where $X$ can rebind after the displacement reaction.

quences such that a single species has a given melting temperature. But, owing to the uncertainty inherent to the model, it is difficult to design a set of sequences for a multi-species system such that all of the melting temperatures are the same. SantaLucia and Turner ${ }^{19}$ quote a $6 \%$ uncertainty on the nearest-neighbor values of the DNA thermodynamic parameters, $\Delta H$ and $\Delta S$. This uncertainty translates to roughly a $3{ }^{\circ} \mathrm{C}$ uncertainty in the melting temperature under standard experimental conditions, which is larger than the width $\left(2{ }^{\circ} \mathrm{C}\right)$ of the entire melting transition. If different pairs of particles within a multi-species system must be annealed at different temperatures, it is impossible to equilibrate the system as a whole.

Here we show that these problems can be overcome by adding free strands of ssDNA that can hybridize to the grafted strands, inhibiting bridge formation. These competing DNA reactions, known as strand-displacement reactions (Figure 1c), have been widely used in the field of DNA nanotechnology to create dynamic and responsive systems. ${ }^{20-23}$ However, in the field of DNAgrafted colloids, strand displacement has, until recently ${ }^{1}$, been employed primarily to melt or change the lattice constants of systems of DNA-grafted nanoparticles. ${ }^{9,14,24,25}$

We will show that the effect of strand displacement is to modify the free energy of hybridization of the grafted DNA strands, which in turn modifies the phase behavior of the particles. The phase behavior of the usual system of particles with complementary grafted strands consists of a single, steep melting transition. Adding a single displacement reaction leads to a broadened melt- ing transition and solid and fluid phases that coexist over a wide range of temperatures. Adding a second displacement reaction leads to two melting transitions: a fluid-to-solid phase transition at low temperature and a solid-to-fluid transition at higher temperature. Confocal microscopy images of systems that show these phase behaviors are shown in Figure 2.

In what follows, we describe the origin of the sharp phase transition for particles grafted with complementary DNA (Section 2) and present a model for predicting this phase behavior for arbitrary sequences and surface concentrations of DNA (Section 3). We then extend this model to show how to overcome the steepness of this transition by adding a single displacement reaction, and we demonstrate the resulting phase behavior in an experimental system (Section 4). We go on to demonstrate further control of the phase behavior through the addition of a second displacement reaction, which yields a second melting transition upon cooling (Section 5). Finally we show more complex phase behavior such as three transitions (Section 6.1) and transitions involving multiple species of particles (Section 6.2). We conclude with a discussion of future directions and practical considerations.

\section{Fundamentals}

Before describing how to use strand displacement reactions to modify the interactions between DNA-grafted particles, we first explain the mechanism of DNA-mediated interactions and why they have such a steep temperature dependence. Our argument follows that of Rogers and Crocker ${ }^{26}$ and Rogers, Shih, and 

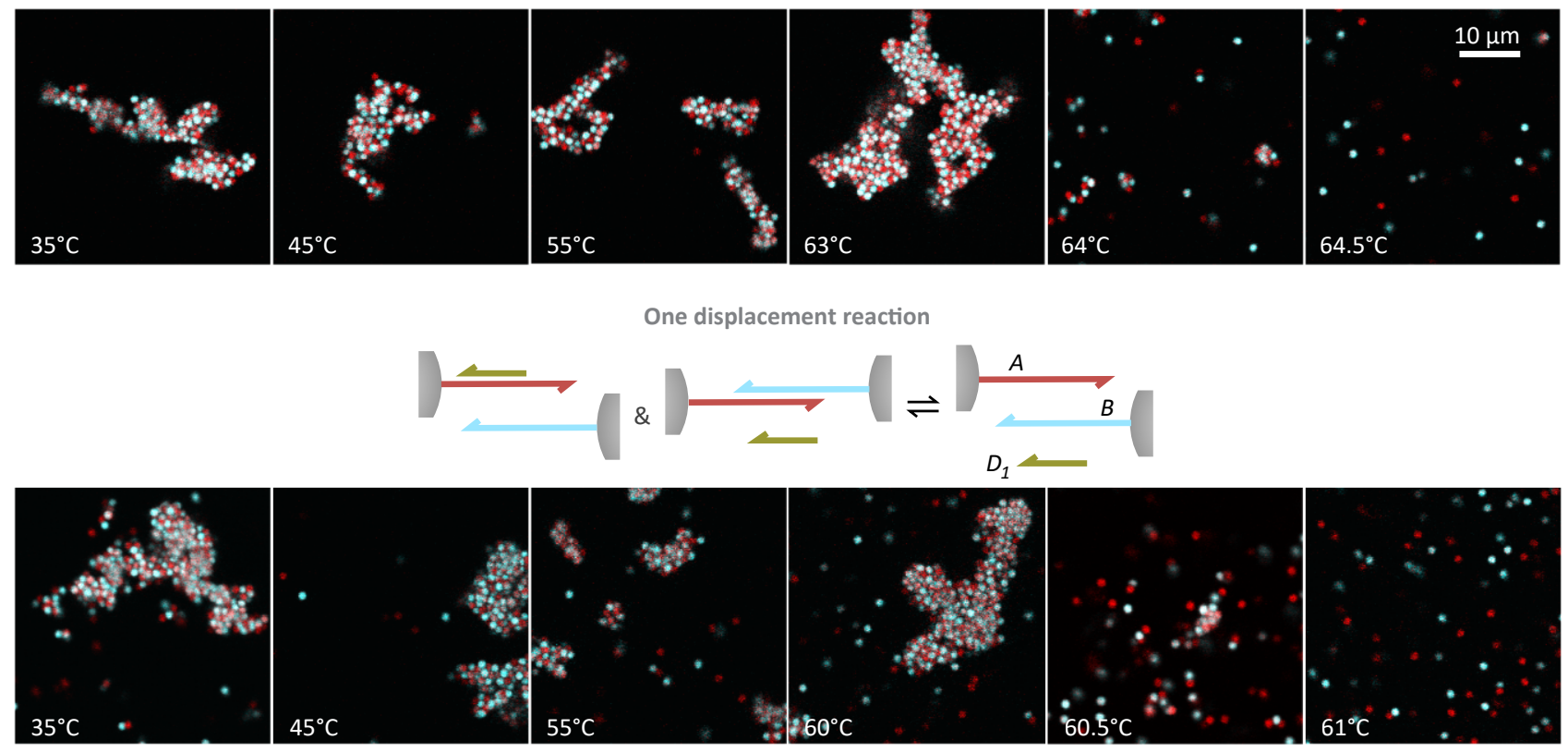

One displacement reaction
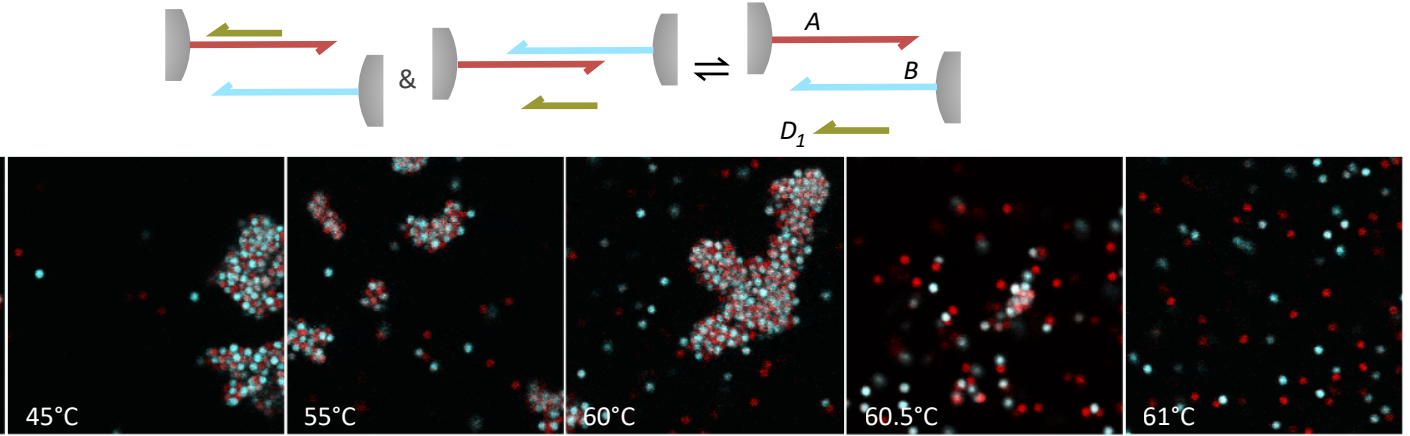

Two displacement reactions
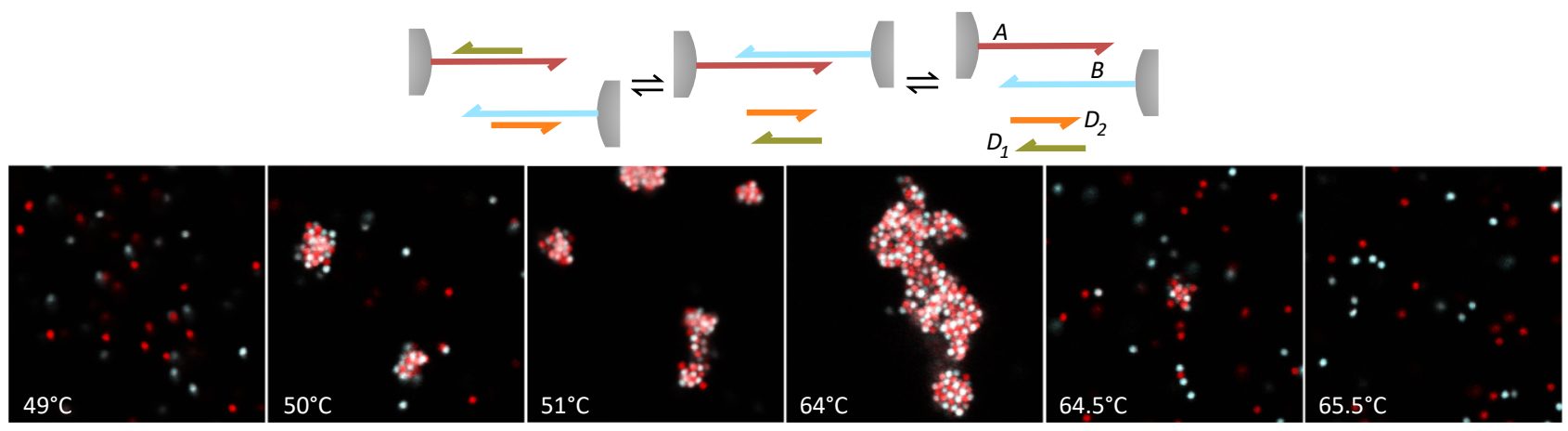

Fig. 2 Fluorescence confocal microscopy images of a 3D system containing two complementary species ( $A$, shown in red, and $B$, shown in blue) of $1-\mu \mathrm{m}$ particles at different temperatures and under different displacement-reaction schemes. These experiments were performed with particles grafted with ssDNA with sequences from Table 3 , with $C_{D_{1}}=1.2 \mu \mathrm{M}$ for the one-displacement-reaction scheme, and with $C_{D_{1}}=C_{D_{2}}=100 \mu \mathrm{M}$ for the two-displacement-reaction scheme. Top: The basic complementary system with no displacing strands. A single steep melting transition occurs in this system. Middle: Adding a single type of displacing strand $\left(D_{1}\right.$, green) introduces a new unbridged state that coexists with the bridged state. As a result, a stable coexistence exists between the solid and fluid phases over a wide range of temperatures. The coexistence is evidenced by the free particles in solution, which are present even at $35^{\circ} \mathrm{C}$. Bottom: Adding another displacing strand $\left(D_{2}\right.$, orange $)$ creates three distinct binding configurations. As a result, a second phase transition emerges. The solid phase is now no longer stable below $50^{\circ} \mathrm{C}$, and it melts upon cooling.

\section{Manoharan ${ }^{5}$.}

To understand the origin of the interparticle interaction in our system, we consider two particles grafted with ssDNA. They attract one another when the ssDNA on one hybridizes with that on the other to form a duplex-or a "bridge" - that links the two particles together

This attraction is mediated by many DNA strands. If the system is not too far from the duplex melting temperature, each of these strands fluctuates between bound and unbound states on timescales short compared to the diffusion time of the particles. Therefore we can treat the attraction as a time-averaged effective interaction, modeled by a free energy $F(r, T) . F(r, T)$ depends on the distance $r$ between the particles and the temperature $T$, as well as on the sequences and concentrations of the DNA strands mediating the binding. $5,15,26$

To illustrate the effect of DNA bridging on the free energy of the system, we consider two identical particles separated by a distance $r$. We calculate the difference in free energy between a state in which the particles are grafted with complementary DNA and a reference state in which they are grafted with non- 
complementary DNA. Then,

$$
\Delta F(r, T)=F_{\text {comp }}-F_{\text {non-comp }}=-k T \ln \left(\frac{Z_{\text {comp }}(r)}{Z_{\text {non-comp }}(r)}\right)
$$

where $k T$ is the thermal energy and $Z(r)$ is the partition function. For the purposes of illustration, we assume that the configurational-conformational entropy of the unbound strands is the same in both the complementary and the non-complementary cases. $^{\dagger}$ Therefore, $Z_{\text {comp }}$ and $Z_{\text {non-comp }}$ count only the ways in which the DNA bridges can form, along with their Boltzmann weights. Because we ignore the entropic contribution, there is only one possible combination of bridges for the noncomplementary particles: no bridges exist $\left(Z_{\text {non-comp }}(r)=1\right)$. Hence, to solve Equation 1 we need only solve for $Z_{\text {comp }}(r)$.

To find the partition function for the complementary case, we begin by writing the probability of finding the pair of complementary DNA-grafted particles in an unbridged state. Because there is only one way to be in an unbridged state, $P_{\text {unbridged }}=1 / Z_{\text {comp }}(r)$, where $Z_{\text {comp }}(r)$ counts all bridged and unbridged configurations. Therefore we can replace $Z_{\text {comp }}(r)$ in Equation 1 by $1 / P_{\text {unbridged, }}$ yielding

$$
\frac{\Delta F}{k T}=\ln P_{\text {unbridged }}
$$

Again for the purposes of illustration, we make another approximation: $P_{\text {unbridged }} \approx(1-\chi)^{N}$, where $\chi$ is the probability of any two complementary strands forming a bridge, and $N$ is the total number of grafted strands that can bind. The assumption behind this approximation is that bridges form independently of one another. Additionally, we assume that $\chi$ and $N$ are constant with $r$ up to some cutoff distance set by the radius of gyration of the ssDNA (see below). With these approximations we find that

$$
\frac{\Delta F}{k T}=\ln (1-\chi)^{N} .
$$

The value of $N$ is related to the surface concentration of the DNA on the particles $\left(C_{i 0}\right)$ and the volume $\left(V_{\text {overlap }}\right)$ in which the binding portions of the two complementary DNA brushes overlap: $N=C_{i 0} V_{\text {overlap }}{ }^{\ddagger}$ The exponent of $N$ in Equation 2 is responsible for the steep transition from a bridged to an unbridged regime as the temperature increases. Because of this exponent, even a small increase in $\chi$, caused by a small change in temperature, leads to a large decrease in the probability that two particles are unbridged.

In this paper, we will use this simplified expression for $\Delta F$, herein referred to as the "simple model," because it captures the essential features of the behavior of the system. One can derive a more accurate expression for the binding energy by accounting for how the density of the binding domains of the DNA strands varies with distance from the particle surface. ${ }^{26}$ To do so, we start by assuming that chemical equilibrium between grafted strands is established locally. We then integrate the density, corrected for

$\dagger$ If we consider this contribution, we would have $Z_{\text {configurational }}=\Omega_{u}$, where $\Omega_{u}$ is the number of configurational-conformational microstates in which the grafted strands do not overlap with each other or with the particles.

$\ddagger$ When the surface concentration of DNA on the two particles is not equal, the smaller of the two concentrations is used to calculate $N$. dependence on distance from the particle surface, over the space between the particles:

$$
\frac{\Delta F}{k T} \approx \int C_{i 0}(r) \ln [1-\chi(r, T)] d r,
$$

where $C_{i 0}(r)$ is the concentration of the binding domains of the grafted DNA strands, $r$ is the spatial coordinate, and $T$ is the temperature. In this more accurate model of the interaction, herein referred to as the "full model," we also consider the repulsive contribution to the interparticle interactions, which arises from the compression of the DNA strands when the particle surfaces come close to one another. This entropic contribution and other details of this model are discussed in reference 26 . In neither the simple nor full models do we assume that the number of bridges is Poisson-distributed, an approximation that has been discussed previously in the literature. ${ }^{26-28}$ However, we do assume independent binding, which is valid when $N$ is large and binding is weak-conditions met in our experimental system.

With an expression for $\Delta F$, from either Equation 2 or Equation 3 , we can predict the singlet fraction $f$-the fraction of particles not bound to any others-as a function of temperature using the equation derived by Dreyfus and coworkers ${ }^{15}$ :

$$
f=\frac{1+2 K C_{p}-\sqrt{1+4 K C_{p}}}{2\left(K C_{p}\right)^{2}}
$$

where $C_{p}$ is the concentration of particles per square micrometer, $K=\left(l^{2} / 4\right) \exp (-z \Delta F(T) / 2 k T), z$ is the average coordination of each particle (for a quasi-2D suspension, $z \approx 3$ ), and $l$ is the range of the interaction. In our system, we take the range to be $15 \mathrm{~nm}$, which is a little bit larger than the radius of gyration of the grafted strands. While previously we made no assumptions about the geometry of our system, this expression for the singlet fraction assumes a quasi-two-dimensional (quasi-2D) suspension of particles. In the remainder of this paper, unless otherwise noted, all discussion and experimental measurements of the singlet fraction pertain to quasi-2D samples. We note, however, that the control over phase behavior achieved using strand-displacement reactions generalizes to three dimensions (3D), as demonstrated in Figure 2.

The expressions given in this subsection are general. They hold for systems containing only grafted strands and for systems containing both grafted and displacing strands. In the next three sections, we examine systems with zero, one, and two displacing strands. In each case, we derive expressions for the probability of hybridization between the DNA strands grafted to the particles, $\chi(T)$, and combine them with Equations 2 and 4 to predict the temperature-dependent singlet fraction.

\section{Displacement-free binding}

To calculate $\chi(T)$ for a pair of particles grafted with complementary DNA in the absence of any strand-displacement reactions, we model the hybridization of complementary DNA sequences $A$ and $B$ as a bimolecular reaction ${ }^{29}$ :

$$
A+B \rightleftharpoons A B .
$$


The equilibrium hybridization yield $\chi=C_{A B} /\left(C_{A}+C_{A B}\right)=$ $C_{A B} / C_{A 0}$ can be evaluated from the equilibrium constant of the reaction and from mass conservation:

$$
\begin{gathered}
K_{\mathrm{eq}}(T)=\frac{C_{A B} C^{\circ}}{C_{A} C_{B}} \\
C_{A 0}=C_{A}+C_{A B} \\
C_{B 0}=b C_{A 0}=C_{B}+C_{A B},
\end{gathered}
$$

where $C^{\circ}=1 \mathrm{M}$ is a reference concentration, $K_{\mathrm{eq}}(T)=$ $\exp \left[-\Delta G_{A B}(T) / k T\right]$ is the equilibrium constant, and $\Delta G_{A B}=$ $\Delta H_{A B}-T \Delta S_{A B}$ is the standard free energy difference between double-stranded $A B$ and single-stranded $A$ and $B$ at the reference concentration $C^{\circ}$. Here $C_{B 0}=b C_{A 0}$, where $b \geq 1$ and $C_{A 0}$ represents the local concentration if $A$ is a grafted strand. We obtain the following equation for the hybridization yield:

$$
\begin{aligned}
\chi & =\frac{C_{A B}}{C_{A 0}} \\
& =\frac{(b+1) K_{e q} \frac{C_{A 0}}{C^{\circ}}+1-\sqrt{(b-1)^{2}\left(K_{e q} \frac{C_{A 0}}{C^{\circ}}\right)^{2}+2(b+1) K_{e q} \frac{C_{A 0}}{C^{\circ}}+1}}{2 K_{e q} \frac{C_{A 0}}{C^{\circ}}},
\end{aligned}
$$

where $\chi$ depends only on $b$ and the concentration-adjusted equilibrium constant $K_{\mathrm{eq}}^{\prime}=K_{\mathrm{eq}} C_{A 0} / C^{\circ}$.

To calculate the equilibrium constant, we use the nearestneighbor model ${ }^{18}$, which gives the concentration-adjusted standard free energy of binding $\Delta G^{\prime}$ :

$$
-\ln K_{\mathrm{eq}}^{\prime}=\frac{\Delta G^{\prime}}{R T}=\frac{\Delta H_{A B}}{R T}-\left[\frac{\Delta S_{A B}}{R}+\ln \left(\frac{C_{A 0}}{C^{\circ}}\right)\right]
$$

where $\Delta H_{A B}$ and $\Delta S_{A B}$ are calculated from the nearest-neighbor model using the actual sequences of the strands. $\S$ The additional entropic term, $\ln \left(C_{A 0} / C^{\circ}\right)$, results from the fact that two single-stranded reactants are consumed upon formation of one double-stranded product. Because $\Delta H_{A B}$ and $\Delta S_{A B}$ are assumed to be constant with temperature ${ }^{18}$, the temperature dependence of $\Delta G^{\prime} / R T$, and thus of Equations 2,3 , and 4, is determined entirely by the enthalpic term, the slope of which can be tuned by changing $\Delta H_{A B}$.

With this expression for $\chi$, we can use Equations 2, 4, 5, and 6 to predict $\Delta G^{\prime} / R T$ and the singlet fraction as a function of temperature, given the sequences (which control $\Delta H_{A B}$ and $\Delta S_{A B}$ ) and the surface concentrations of the grafted strands. By choosing values of these parameters that are typical for our experimental systems (described in Sections 4.2, 5.2, and 6) we can use our model to explore the phase behavior over a wide range of conditions. In Figure 3, we see that changing the surface concentration of DNA shifts $\Delta G^{\prime}$ such that particles with higher surface concentrations melt at higher temperatures. This result has been validated experimentally in previous work. ${ }^{15,30}$ Modifying $\Delta H_{A B}$

$\S$ Here we switch to the Gibbs free energy instead of the Helmholtz free energy; while before we considered two particles held a fixed distance apart, now we consider a system of many components free to explore space. and $\Delta S_{A B}$ changes the slope of $\Delta G^{\prime}$, which also shifts the melting transition but does not significantly change its steepness. This effect-shifting the curve without changing the steepness-can be seen in the melting curves at zero displacing-strand concentration in Figures 4 and 5 and the blue curve in Figure 7. In each of these systems, the grafted strands have different sequences and therefore different $\Delta H_{A B}$ and $\Delta S_{A B}$.

The plots in Figure 3 show that it is difficult to control the steepness of the melting transition in a displacement-free system. One can adjust the sequences of the grafted strands and their surface concentrations, but both of these parameters affect the singletfraction curves in a similar way: they shift the melting transition, but neither changes the steepness significantly. Although it is possible to reduce the steepness by using very low DNA surface concentrations, the DNA strands would need to bind strongly to keep the melting transition at a similar temperature. In this regime, the strands no longer fluctuate between bridged and unbridged configurations on timescales short compared to particle motion, and non-equilibrium behavior can result. ${ }^{30,31}$

Fundamentally, what limits control over the shape of the melting transition is the linear dependence of $\Delta G^{\prime}(T)$ on temperature. Changing the sequence or surface concentration can only change its slope. To control the shape of the melting transition we need to make $\Delta G^{\prime}(T)$ a nonlinear function of temperature. In the following sections, we show how to do this by adding free strands that displace the grafted strands in a controlled way.

\section{Single strand-displacement reaction}

To increase the range of temperatures over which the system can equilibrate, we must significantly decrease the slope of $\Delta G^{\prime}(T)$ near the melting transition. As shown in the previous section, this cannot be accomplished simply by modifying the grafted sequences or their surface concentrations. Instead, we introduce a competing interaction that effectively decreases the attraction between the two particles as the temperature decreases.

We create this competing interaction by introducing a freely dissolved displacing strand $D_{1}$ that can bind to one of the grafted strands $(A)$. By binding $A$, these free strands can displace the hybridized $A B$ pairs, causing the particles to unbind, while maintaining the same overall number of DNA duplexes (see diagram in the middle panel of Figure 2). As a result, the melting transition broadens such that coexistence between solid and fluid phases persists over a wide range of temperatures, as shown in the confocal micrographs in the middle panel of Figure 2.

\subsection{Modeling the single strand-displacement reaction}

To predict the singlet fraction of this modified system as a function of temperature, we again derive an expression for $\chi(T)$. We take into account the effect of a single displacement reaction on the probability that DNA bridges will form between the particles.

As in Section 3, we begin by writing down the equilibrium reactions for our system, now considering both the particle bridging 

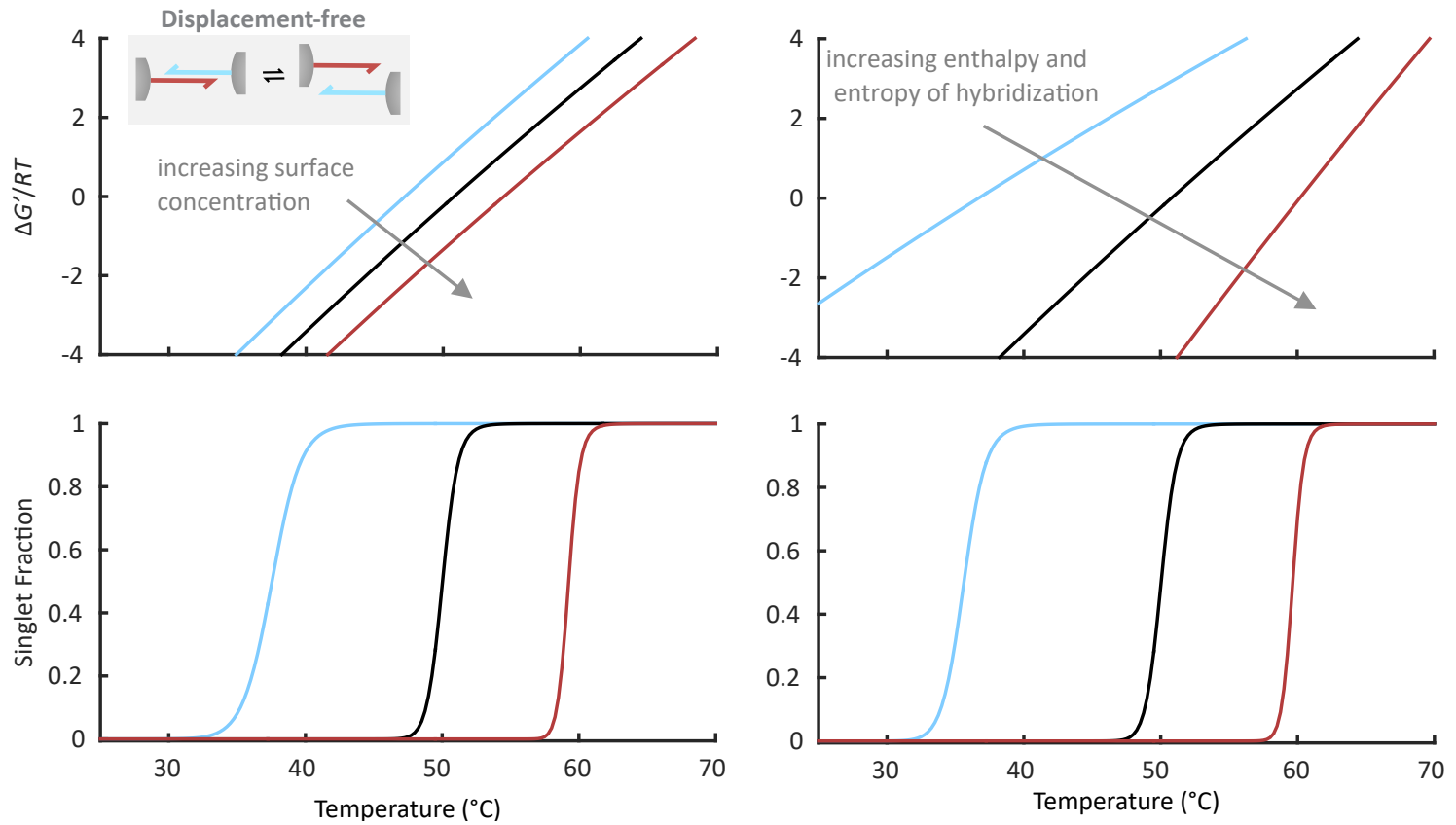

Fig. 3 Temperature-dependent behavior of particles grafted with complementary DNA (see cartoon inset). Left: Plots of $\Delta G^{\prime}$ (top) and singlet fraction (bottom) as a function of temperature and DNA surface concentration, calculated using Equations 2, 4, 5, and 6 . The calculations are performed for DNA surface concentrations $C_{A 0}=76.7 \mu \mathrm{M}$ (blue curves), $230 \mu \mathrm{M}$ (black), and $690 \mu \mathrm{M}$ (red). For all three curves the hybridization enthalpy and entropy of the DNA are $\Delta H_{A B}=-63.8 \mathrm{kcal} / \mathrm{mol}$ and $\Delta S_{A B}=-180.3 \mathrm{cal} / \mathrm{mol} / \mathrm{K}$. Right: Plots of the same values, but now for constant surface concentration $\left(C_{A 0}=230 \mu \mathrm{M}\right)$ and varying enthalpy and entropy of DNA hybridization: $\Delta H_{A B}=-44.66 \mathrm{kcal} / \mathrm{mol}$ and $\Delta S_{A B}=-126.21 \mathrm{cal} / \mathrm{mol} / \mathrm{K}$ (blue curves), $\Delta H_{A B}=-63.8 \mathrm{kcal} / \mathrm{mol}$ and $\Delta S_{A B}=-180.3 \mathrm{cal} / \mathrm{mol} / \mathrm{K}$ (black), and $\Delta H_{A B}=-95.7 \mathrm{kcal} / \mathrm{mol}$ and $\Delta S_{A B}=-270.45 \mathrm{cal} / \mathrm{mol} / \mathrm{K}(\mathrm{red})$. Note that changing either surface concentration or the enthalpy and entropy of hybridization shifts the melting transition, but does not significantly change its steepness. This calculation shows that sequence design and grafting density offer limited control over the shape of the melting transition.

and the displacement processes:

$$
\begin{gathered}
A+B \rightleftharpoons A B \\
A B+D_{1} \rightleftharpoons A D_{1}+B .
\end{gathered}
$$

We derive the hybridization yield needed to complete Equation 2 from the equilibrium constant and conservation of mass, assuming that the free strand $D_{1}$ is in large excess:

$$
\begin{gathered}
K_{e q}^{(1)}(T)=\frac{C_{A B} C^{\circ}}{C_{A} C_{B}} \\
K_{e q}^{(2)}(T)=\frac{C_{A D_{1}} C_{B}}{C_{A B} C_{D_{1}}} \\
C_{A 0}=C_{A}+C_{A B}+C_{A D_{1}} \\
C_{B 0}=b C_{A 0}=C_{B}+C_{A B} \\
C_{D_{1} 0} \approx C_{D_{1}},
\end{gathered}
$$

where $K_{e q}^{(1)}$ is the equilibrium constant for the reaction shown in Equation 7 and $K_{e q}^{(2)}$ for Equation 8, and $C_{D_{1}}$ is the free concen-

|| Because the grafted DNA strands are confined roughly within a spherical shell with volume $V_{\text {shell }} \approx 4 \pi a^{2} L$, this condition is satisfied so long as $C_{D_{1} 0} / C_{A 0} \gg \phi /(1-$ $\phi) V_{\text {shell }} / V_{\text {particle }}=3 \phi /(1-\phi) L / a$, where $\phi$ is the colloid volume fraction, $a$ is the particle radius, and $L \approx 11 \mathrm{~nm}$ is the thickness of the DNA brush, which is taken to be the same as the radius of gyration of the DNA. For our experimental conditions, $3 \phi /(1-\phi) L / a \approx 0.005$. tration of $D_{1}$ in the buffer in which the particles are suspended. The hybridization yield $\chi=C_{A B} /\left(C_{A}+C_{A B}+C_{A D_{1}}\right)=C_{A B} / C_{A 0}$ can then be expressed in the same form as Equation 5 ,

$$
\chi=\frac{(b+1) K_{e q}^{\prime}+1-\sqrt{(b-1)^{2}\left(K_{e q}^{\prime}\right)^{2}+2(b+1) K_{e q}^{\prime}+1}}{2 K_{e q}^{\prime}},
$$

by redefining $K_{e q}^{\prime}$ in the following way:

$$
-\ln K_{e q}^{\prime}=\frac{\Delta G^{\prime}}{R T}=-\ln \left[\frac{K_{e q}^{(1)} C_{A 0} / C^{\circ}}{1+K_{e q}^{(1)} K_{e q}^{(2)} C_{D_{1} 0} / C^{\circ}}\right] .
$$

Thus we arrive at the same model as the one without displacing strands (Section 3), but with a modified expression for $\Delta G^{\prime}(T)$.

We can understand how the displacement reaction modifies the temperature dependence of the interaction by examining limiting cases. In the high-temperature limit, where $K_{e q}^{(1)} K_{e q}^{(2)} C_{D_{1} 0} / C^{\circ} \ll$ 1, $\Delta G^{\prime}$ reduces to Equation 6 . Thus, at high temperatures the displacement reaction has no effect on bridge formation-or, in fact, on the system at all. It can therefore be ignored.

In the opposite, low-temperature limit we find

$$
\frac{\Delta G^{\prime}}{R T} \stackrel{\text { low } T}{\longrightarrow} \frac{\left(\Delta H_{A B}-\Delta H_{A D_{1}}\right)}{R T}-\left[\frac{\left(\Delta S_{A B}-\Delta S_{A D_{1}}\right)}{R}+\ln \left(\frac{C_{D_{1} 0}}{C^{\circ}}\right)\right] .
$$

In this limit we see that the displacement reaction simply lowers the free energy difference between the bridging and non-bridging configurations. As a result, if the grafted and displacing strands 
are designed to have the same enthalpy change upon hybridization (that is, $\Delta H_{A B}=\Delta H_{A D_{1}}$ ),

$$
\frac{\Delta G^{\prime}}{R T} \stackrel{\text { low } T}{\longrightarrow}-\left[\frac{\left(\Delta S_{A B}-\Delta S_{A D_{1}}\right)}{R}+\ln \left(\frac{C_{D_{1} 0}}{C^{\circ}}\right)\right] .
$$

When $\Delta H_{A B}=\Delta H_{A D_{1}}$, we expect the melting transition to be broadened, since Equation 11 tells us that the free energy must become independent of temperature at low temperature. Therefore, adding the displacing strand makes the free energy of binding $\Delta G^{\prime}(T)$ a nonlinear function of temperature.

The result of this nonlinearity in $\Delta G^{\prime}(T)$ is a melting transition that can be tuned from steep to flat. To tune the transition, one can adjust either the sequences of the strands, which control $\Delta H_{A B}, \Delta H_{A D_{1}}, \Delta S_{A B}$, and $\Delta S_{A D_{1}}$, or the displacing-strand concentration $C_{D_{1}}$. Theoretical predictions of this behavior, obtained by inserting Equations 9 and 10 into our free-energy and singletfraction equations from Section 2, are shown by the curves in Figure 4. Experimental results are shown in the following subsection.

\subsection{Experiments using a single strand-displacement reac- tion}

The wide region of coexistence between the solid and fluid phases that results from introducing a single displacing strand can be seen in the middle panel of Figure 2, which shows confocal micrographs of a 3D suspension of two species of fluorescently dyed particles grafted with sequences $A$ (blue) and $B$ (red) and mixed with displacing strand $D_{1}$. The percent of unbound particles in this system remains non-zero and constant from 35 to $60^{\circ} \mathrm{C}$. Although in these experiments we have not waited long enough for nucleation of crystals to occur, our previous study ${ }^{1}$ showed that, at longer times, crystals can form over a wide range of temperatures.

To generate DNA sequences that result in this wide range of coexistence, we follow a set of design rules intended to minimize the melting temperatures of secondary structures and the crosstalk between sequences (see Section 8.1). We then select sequences with melting temperatures between 40 and $65{ }^{\circ} \mathrm{C}$ and for which $\Delta H_{A B}$ and $\Delta H_{A D_{1}}$ fall within $5 \%$ of one another, so that Equation 11 applies. We then use Equations 2, 4, 9, and 10 to predict the 2D phase behavior and verify that the sequences produce the desired effect. The resulting sequences are shown in Table 1, and their thermodynamic parameters are given in Table 2.

Table 1 DNA sequences used for single strand-displacement experiments

\begin{tabular}{|c|c|}
\hline Strand name & Base Sequence \\
\hline $\bar{A}$ & $5^{\prime}-\left(T_{51}\right)-$ GAGTTGCGGTAGAC - $3^{\prime}$ \\
\hline$B$ & $5 '-\left(T_{51}\right)-$ AATGCCTGTCTACC -3' \\
\hline$D_{1}$ & 5'-ACCGCAA-3' \\
\hline
\end{tabular}

As predicted by our model, the location and steepness of the melting transition and degree of coexistence can be tuned by changing the concentration, $C_{D_{1}}$, of the displacing strand in solution (Figure 4). According to Equation 11, increasing the concentration of displacing strand should favor unbridged configurations
Table 2 Thermodynamic parameters for single-displacement sequences used. The enthalpy and entropy of hybridization are calculated using the NUPACK software ${ }^{32}$ for $50 \mathrm{mM} \mathrm{NaCl}$ and $10 \mathrm{mM} \mathrm{MgCl}_{2}$.

\begin{tabular}{lll}
\hline Duplex name & $\Delta H(\mathrm{kcal} / \mathrm{mol})$ & $\Delta S(\mathrm{cal} / \mathrm{mol} / \mathrm{K})$ \\
\hline$A B$ & -56.6 & -163.0 \\
$A D_{1}$ & -53.6 & -149.1 \\
\hline
\end{tabular}

over bridged configurations at low temperature. Our experiments on quasi-2D samples (described in detail in Section 8.4) verify this prediction. With no displacing strands, the system behaves as a simple complementary reaction: the singlet fraction rapidly increases to unity as the temperature increases. As the concentration of displacing strands is increased to $6 \mu \mathrm{M}$, a $10{ }^{\circ} \mathrm{C}$-wide region of coexistence emerges between a solid phase containing about $25 \%$ of particles and a fluid phase containing the remainder. At higher concentrations of displacing strands we observe a fluid at all temperatures. Thus, the low-temperature equilibrium singlet fraction can be tuned between zero and one through a small change in the concentration of displacing strand, and the width of the melting transition can be varied from 2 to at least $10{ }^{\circ} \mathrm{C}$.

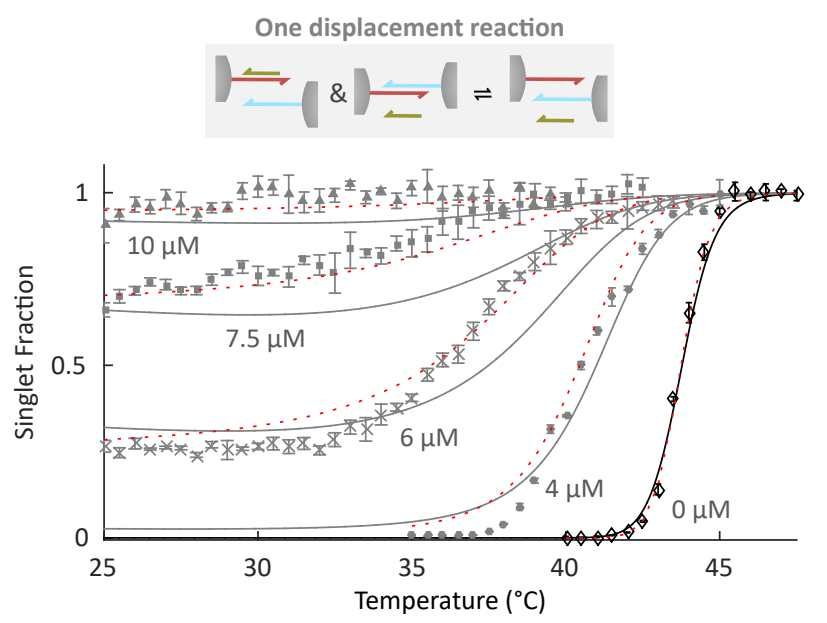

Fig. 4 In an experimental system with one displacement reaction, changing the concentration of displacing strands can alter the width of the melting transition and the low-temperature singlet fraction. The plot shows the experimental singlet fraction (symbols) for different displacing-strand concentrations (from right to left, $C_{D_{1}}=0,4,6,7.5$, and $10 \mu \mathrm{M}$ ). Fits of the model from Section 4.1 calculated using Equations 2, 4, 9, and 10 are shown as solid lines. For comparison, fits of the full model, calculated using Equation 3 instead of Equation 2 and including the interparticle repulsion, are shown as red dashed lines. Both the experiments and theory correspond to 6500 DNA strands per particle. The error bars correspond to the standard deviation from three measurements.

As shown in Figure 4, both the simplified model (shown as solid lines on the figure) and the full model (shown as dashed lines) detailed in Sections 2 and 4.1 capture the observed behavior of the system at different displacing-strand concentrations. To check the validity of our models, we fit them to the data using four free parameters $\left(\Delta H_{A B}, \Delta S_{A B}, \Delta H_{A D_{1}}\right.$, and $\left.\Delta S_{A D_{1}}\right)$, as described in Section 8.7. The fits for these four parameters are performed simultaneously for all displacing-strand concentrations. For the simplified model, the best-fit parameters are all within $8 \%$ of those 
predicted by NUPACK. For the full model the parameters are all within $3 \%$ of the NUPACK predictions. The agreement shows that both models can be used, in conjunction with theoretical calculations of the thermodynamic parameters, to predict the phase behavior in the presence of displacing strands. Although the full model fits the experimental data much better than does the simplified model, the simplified model is, nonetheless, a useful tool for designing DNA strands that will produce a particular behavior and is considerably easier to implement.

\section{Two strand-displacement reactions}

By adding a second displacing strand to the same solution we can make the free energy of binding $\Delta G^{\prime}(T)$ not only nonlinear, but non-monotonic as well. This effect occurs if the second displacing strand $D_{2}$ can bind to the grafted strand $B$ (see the diagram in the bottom panel of Figure 2). The result of this competition for binding between the various DNA strands is to invert the dependence on temperature, such that the interparticle attraction strengthens with increasing temperature over a range of temperatures before falling again at high temperatures. The effects of this inverted temperature dependence can be seen in the confocal micrographs in the bottom panel of Figure 2 .

\subsection{Modeling two strand-displacement reactions}

To model the effects of two strand-displacement reactions, we begin with the set of reactions

$$
\begin{gathered}
A+B \rightleftharpoons A B \\
A B+D_{1} \rightleftharpoons A D_{1}+B \\
A B+D_{2} \rightleftharpoons A+B D_{2} .
\end{gathered}
$$

We assume that both $D_{1}$ and $D_{2}$ are in large excess, such that

$$
\begin{gathered}
K_{e q}^{(1)}(T)=\frac{C_{A B} C^{\circ}}{C_{A} C_{B}} \\
K_{e q}^{(2)}(T)=\frac{C_{A D_{1}} C_{B}}{C_{A B} C_{D_{1}}} \\
K_{e q}^{(3)}(T)=\frac{C_{B D_{2}} C_{A}}{C_{A B} C_{D_{2}}} \\
C_{A 0}=C_{A}+C_{A B}+C_{A D_{1}} \\
C_{B 0}=b C_{A 0}=C_{B}+C_{A B}+C_{B D_{2}} \\
C_{D_{1} 0} \approx C_{D_{1}} \\
C_{D_{2} 0} \approx C_{D_{2}} .
\end{gathered}
$$

To calculate the hybridization yield of bridge formation between the particles, $\chi=C_{A B} /\left(C_{A}+C_{A B}+C_{A D_{1}}\right)=C_{A B} / C_{A 0}$, we first need an expression for the concentration-adjusted free energy. For the reaction network given by Equation 12,

$$
\frac{\Delta G^{\prime}}{R T}=-\ln \left[\frac{K_{e q}^{(1)} C_{A 0} / C^{\circ}}{\left(1+K_{e q}^{(1)} K_{e q}^{(2)} C_{D_{1} 0} / C^{\circ}\right)\left(1+K_{e q}^{(1)} K_{e q}^{(3)} C_{D_{2} 0} / C^{\circ}\right)}\right] .
$$

We can then calculate the concentration-adjusted equilibrium constant using $\ln K_{e q}^{\prime}=-\Delta G^{\prime} / R T$ and substitute into Equation 9 to find $\chi$.

In the high-temperature limit, $\Delta G^{\prime}$ again reduces to Equation 6 when the concentration of the two displacing strands are equal $\left(C_{D_{1} 0}=C_{D_{2} 0}=C_{D 0}\right)$ and when the hybridization free energies are the same for the bridging and displacing strands $\left(\Delta G_{A B}=\right.$ $\left.\Delta G_{A D_{1}}=\Delta G_{B D_{2}}\right)$. In the opposite, low-temperature, limit we find

$$
\frac{\Delta G^{\prime}}{R T} \stackrel{\text { low } T}{\longrightarrow}-\frac{\Delta H_{A B}}{R T}+\left[\frac{\Delta S_{A B}}{R}+\ln \left(\frac{C_{A 0}}{C^{\circ}}\right)+2 \ln \left(\frac{C_{D 0}}{C_{A 0}}\right)\right] .
$$

Note that the sign of the first term, $\Delta H_{A B} / R T$, is inverted from that in Equation 6. In other words, the free energy difference between bridging and non-bridging configurations actually becomes larger as the temperature decreases, making bridge formation less favorable.

As a result, the singlet fraction shows two transitions: a freezing transition from fluid to solid that occurs upon heating from low temperature, and a melting transition from solid to fluid upon further heating. These transitions can be understood from the molecular-scale interactions shown in the diagram at the top of Figure 5. At low temperatures, enthalpy favors two displacing strands binding to two grafted strands, which has more base pairs than two grafted strands binding together. As a result, the grafted strands are unavailable to form bridges, the particles do not attract one another, and the system remains in a fluid phase. On heating, the displacing strands can detach from the grafted strands. Although the enthalpy of this state is higher than that of the low-temperature state since there are fewer base pairs, this enthalpic increase is compensated by the increase in entropy caused by the liberation of the two displacing strands. As a result, the grafted strands are free to form bridges, and the particles can form a solid phase. The solid phase is therefore entropically stabilized, and the range of temperatures over which it is stable can be tuned by changing the concentration of the displacing strands, as predicted by the model. Finally, at high temperature the grafted strands dissociate, and the solid phase melts.

As in the single displacement-reaction case, we can theoretically predict the behavior of the system by solving for $\chi$ as described above and then using Equation 2 to calculate $\Delta F$. Finally, we substitute this value of $\Delta F$ into Equation 4 to solve for the singlet fraction. The resulting theoretical singlet fraction curves are shown by the solid lines in Figure 5. The experimental realization of this system is described in the following subsection.

\subsection{Experiments using two strand-displacement reactions}

The appearance of a second phase transition in the two displacement reaction system is shown experimentally in the bottom panel of Figure 2. This figure shows confocal images from a 3D system containing two species of particles $(A$ and $B$ ) and two displacing strands $\left(D_{1}\right.$ and $\left.D_{2}\right)$. The concentrations of the two displacing strands are equal. Our experiment shows that at $65.5^{\circ} \mathrm{C}$ the entire system is in a fluid state that, on cooling, transitions to a solid at $64^{\circ} \mathrm{C}$. This solid phase persists to $51^{\circ} \mathrm{C}$, at which point the system melts on further cooling, so that at $49^{\circ} \mathrm{C}$ it is once again fully in the fluid state. 
Table 3 DNA sequences used for experiments with two stranddisplacement reactions

\begin{tabular}{ll}
\hline Strand name & Base Sequence \\
\hline$A$ & 5'-(T54)-CTAACTGCGGT-3' \\
$B$ & 5'-(T, T $_{54}$-CTTACCGCAGT-3' \\
$D_{1}$ & GCAGTTAG \\
$D_{2}$ & GCGGTAAG \\
\hline
\end{tabular}

Table 4 Thermodynamic parameters for sequences used for two displacement reactions, calculated using the NUPACK software for $250 \mathrm{mM}$ $\mathrm{NaCl}$.

\begin{tabular}{lll}
\hline Duplex name & $\Delta H(\mathrm{kcal} / \mathrm{mol})$ & $\Delta S(\mathrm{cal} / \mathrm{mol} / \mathrm{K})$ \\
\hline$A B$ & -63.6 & -173.7 \\
$A D_{1}$ & -64.4 & -186.1 \\
$B D_{2}$ & -69.4 & -195.7 \\
\hline
\end{tabular}

We design our sequences in the same way as for the single displacement scheme, but with the constraint that the free energies of hybridization are all equal $\left(\Delta G_{A B}=\Delta G_{A D_{1}}=\Delta G_{B D_{2}}\right)$ to within $5 \%$. The sequences are shown in Table 3 and their thermodynamic parameters in Table 4. With this constraint, we can control the width of the solid region in the phase diagram by changing the concentration of the two displacing strands jointly $\left(C_{D_{1}}=C_{D_{2}}\right)$, as predicted by Equation 14 and shown in the quasi$2 \mathrm{D}$ measurements in Figure 5. Increasing the concentration of displacing strands favors the fluid phase, where more displacing strands are bound, over the solid phase.

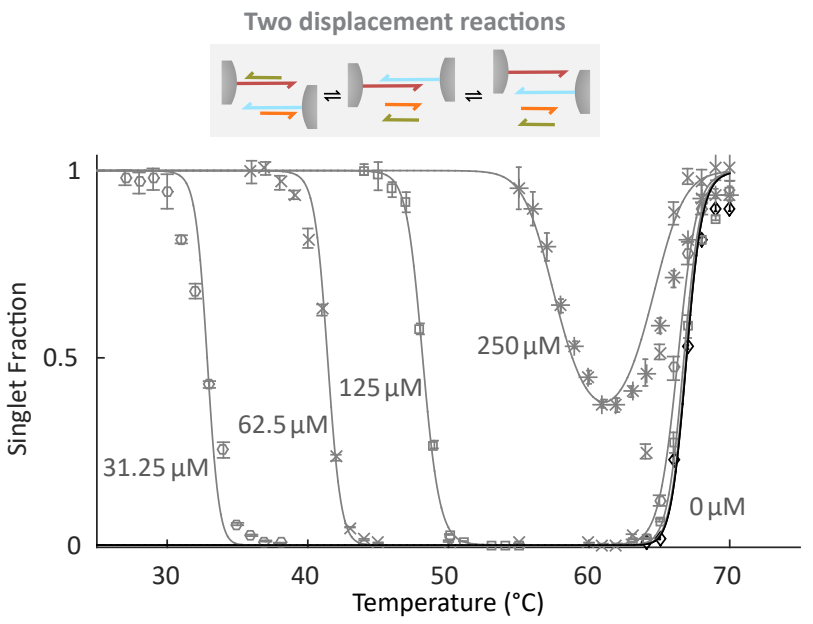

Fig. 5 In an experimental system with two displacement reactions, changing the concentration of displacing strands alters the width (in temperature) of the regime where the solid phase is stable. The experimentally measured singlet fractions are shown by the symbols, where each type of symbol represents a different displacing-strand concentration. The solid lines represent fits of the model from Section 5.1 using Equations 2, 4, 9, and 13. In both the experiments and the fits, there are 6500 DNA strands per particle. Error bars denote the standard deviation from three measurements.

This tunability is shown experimentally in Figure 5. At low concentration of displacing strand $(31 \mu \mathrm{M})$ there is a $30^{\circ} \mathrm{C}$-wide solid regime between two fluid phases. At higher concentration of displacing strand, the width of this regime decreases, so that at $125 \mu \mathrm{M}$ it is approximately $13{ }^{\circ} \mathrm{C}$ wide. At $250 \mu \mathrm{M}$ of displacing strands there is no temperature at which the system is completely solid. Thus, the minimum width of a regime in which the sample is fully solid is about $10^{\circ} \mathrm{C}$.

Our simplified model, shown as solid lines in the figure, again captures the behavior of our experimental measurements. We fit the model to the data, allowing $\Delta H_{A B}, \Delta H_{A D_{1}}$, and $\Delta H_{B D_{2}}$ to vary while holding $\Delta S_{A B}, \Delta S_{A D_{1}}$, and $\Delta S_{B D_{2}}$ at the values calculated with NUPACK. The values returned by each of the fits are within $3.3 \%$ of their NUPACK values, within the uncertainty of calculations based on the nearest-neighbor model (which NUPACK uses). Again, the agreement validates the use of the model as a tool for designing DNA sequences to yield prescribed phase behaviors.

\section{Combining displacement-free and displacement-mediated interactions}

Having shown that our model explains and predicts the experimentally observed phase behavior in both the one- and twodisplacement-reaction schemes, we now show how to combine these schemes to design more complex behavior. We show that with careful design of the DNA sequences, so as to remove crosstalk between non-complementary sequences, the different strand-displacement reactions can operate independently in the same solution. Thus, multiple such reactions can be combined to create multiple transitions at different temperatures between the same pair of particles, or to create independent phase transitions between multiple species of particles in the same solution.

\subsection{Three phase transitions}

The different interactions described in the previous sections can be combined to create more complex interactions by grafting multiple strands of DNA to each particle. Here we demonstrate a system in which there are three transitions between fluid and solid phases. We create this behavior by combining a transition at low temperature, which arises from the reaction of grafted strands only, with freezing and melting transitions at higher temperatures, which arise from a two-displacement-reaction scheme. Our system consists of two particle species, depicted as gray and red in Figure 6a.

Realizing this set of transitions requires four grafted DNA strands ( $5 A$ and $5 C$ on red and $5 B$ and $5 E$ on gray) and two displacing strands $\left(5 D_{1}\right.$ and $\left.5 D_{2}\right)$, as shown in Figure $6 \mathrm{a}$ and Table 5 . The thermodynamic parameters are given in Table 6 . We design the sequences for $5 A, 5 B, 5 D_{1}$, and $5 D_{2}$ following the protocol described in Section 5.2, so as to place the high-temperature melting transition near $55^{\circ} \mathrm{C}$. We make strands $5 C$ and $5 E$ short compared to $5 A$ and $5 B$ so that the melting transition caused by this interaction is at a low enough temperature $\left(30^{\circ} \mathrm{C}\right)$ to leave room for a third transition between the two. To allow the two sets of transitions (displacement-free and two-displacement) to occur independently of one another, we design the DNA sequences so that they do not contain any complementary domains across the two sets of reactions, as discussed in Section 8.1. Here, one set involves the sequences $5 A, 5 B, 5 D_{1}$, and $5 D_{2}$, and the other involves $5 C$ and $5 E$. Finally, we tune the location of the third transition (a freezing transition) by changing the concentration of displacing 
a
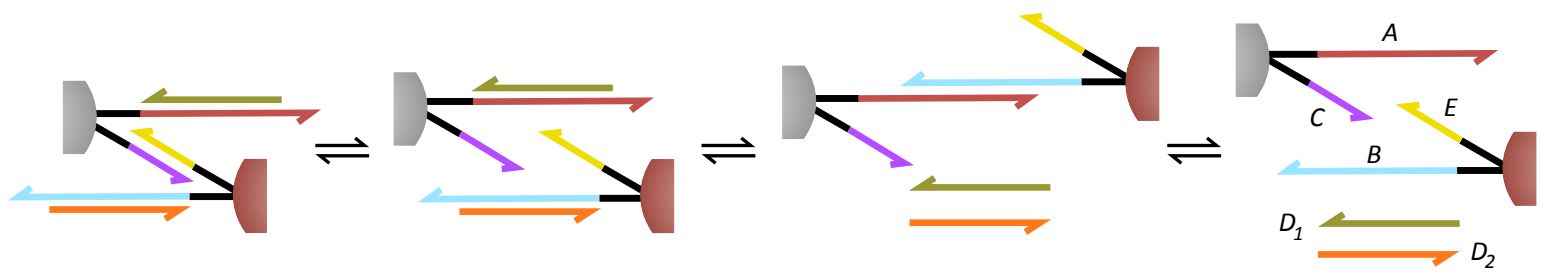

b

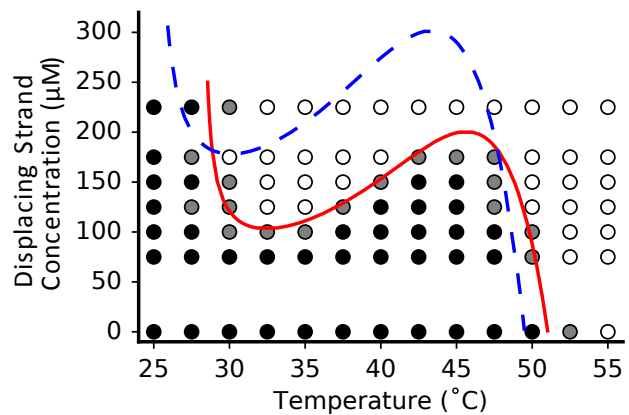

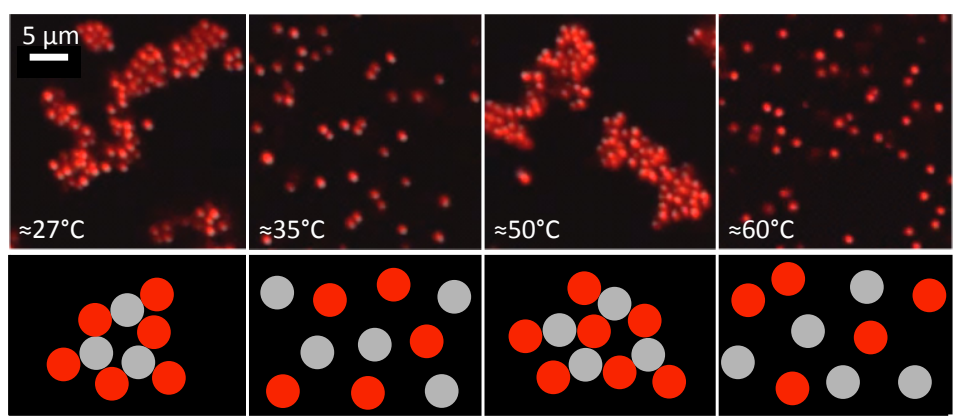

Fig. 6 Demonstration of a system that shows three phase transitions (solid to fluid to solid to fluid). There are two types of particles, one identified by red fluorescent dye and the other undyed (shown in gray on diagrams). a) Schematic of the DNA and particles. The combination of a low-meltingtemperature interaction between complementary grafted strands and a two-displacing-strand-mediated interaction creates the three phase transitions. b) Phase diagram showing the state of aggregation of a 3D suspension of the particles as a function of temperature and displacing-strand concentration $C_{D 1}=C_{D 2}$. The black symbols indicate an aggregated state (less than approximately $15 \%$ of particles unbound), the white symbols indicate a fluid state (more than approximately $75 \%$ of particles unbound), and the grey symbols indicate that the system is between these two bounds. The red line shows the best fit of a model for the phase boundary, which represents the displacing-strand concentration at which the singlet fraction is $50 \%$ for each temperature. The dashed blue line shows this same phase boundary using the nearest-neighbor values for the thermodynamic parameters instead of the best-fit values. c) Fluorescence confocal images of this system with displacing-strand concentration $C_{D 1}=C_{D 2}=125 \mu \mathrm{M}$. As shown in the schematic below the micrographs, the aggregated states occur when the dyed and undyed particles interact with one another, and the fluid states occur when there are no interactions. The temperatures given for the micrographs are approximate.

strands in the solution.

The phase behavior of the resulting experimental system agrees with the predictions of a model for the phase boundary, as shown in Figure 6b. Here we measure the experimental phase behavior for a 3D sample as a function of displacing-strand concentration and temperature, and we compare to the predictions of a model that combines the elements of Sections 3 and 5.1.

To model the behavior of this combined system, we solve for the hybridization probability of $A$ and $B, \chi_{A B}=C_{A B} / C_{A 0}$, using Equations 9, 13, and the relation $\ln K_{e q}^{\prime}=-\Delta G^{\prime} / R T$. We solve for the hybridization probability of $C$ and $E, \chi_{C E}=C_{C E} / C_{E 0}$, using Equations 5 and 6 . We then calculate the singlet fraction using Equation 4, where the binding strength is given by

$$
\frac{\Delta F}{k T}=\ln \left(1-\chi_{A B}\right)^{N_{A}}+\ln \left(1-\chi_{C E}\right)^{N_{E}}
$$

where $N_{i}$ is related to the surface concentration of DNA of type $i$, as discussed in Section 2.

We use our simple 2D model to fit a theoretical phase boundary to the data using three parameters, $f_{1}, f_{2}$, and $f_{3}$, which are coefficients for the nearest-neighbor thermodynamic parameters: $f_{1} \Delta H_{A B}, f_{1} \Delta S_{A B}, f_{2} \Delta H_{A D_{1}}, f_{2} \Delta S_{A D_{1}}, f_{2} \Delta H_{B D_{2}}, f_{2} \Delta S_{B D_{2}}, f_{3} \Delta H_{C E}$, $f_{3} \Delta S_{C E}$. The $f_{i}$ therefore characterize the deviation from nearestneighbor predictions. Section 8.7 provides a detailed description of the fitting method. Given the best-fit coefficients, we then calculate the theoretical phase boundary by calculating the displacing-strand concentration at which the singlet fraction is 0.5 for a range of temperatures from 25 to $55^{\circ} \mathrm{C}$. This phase boundary is shown as the red curve in Figure 6b.

The fits show that the actual thermodynamic parameters are within $11 \%$ of the nearest-neighbor values, which are given in Table 6 . The agreement is good, considering that the the measurements were performed on a 3D sample and compared to a singlet fraction model developed for quasi-2D systems. By comparison, the phase boundary calculated using our model and the nearestneighbor values (instead of the best-fit values) has the same shape but is shifted slightly toward decreasing temperature and higher displacing-strand concentration (blue curve in Figure 6b). Therefore, the $11 \%$ uncertainty in the predictions for the thermodynamic parameters translates to an uncertainty of about a factor of two in the displacing-strand concentration required to achieve a given phase behavior.

Nonetheless, the simple model (Equation 15) with the nearestneighbor predictions of the thermodynamic parameters still proves useful in design. To demonstrate, we use the model to design a system that shows solid-fluid-solid-fluid phase behavior, where the transitions are spaced equally as a function of temperature. Using the nearest-neighbor parameters in our model, we predict that the displacing-strand concentration should be less than $250 \mu \mathrm{M}$ to satisfy these constraints. We then vary the concentration of displacing strands until we realize the desired behavior. In the example shown in Figure $6 c$, we find that the experimental system at $125 \mu \mathrm{M}$ shows an interior fluid-solid transition that occurs about $10{ }^{\circ} \mathrm{C}$ above the low-temperature solid-fluid 
transition and about $15{ }^{\circ} \mathrm{C}$ below the high-temperature solidfluid transition, close to our design goals. Although the design process requires some experimental tuning, the model provides a good starting point. Also, the tuning does not require varying the sequences, only the displacing-strand concentrations-which are easy to vary, as we discuss in Section 7.

Table 5 DNA sequences used for a system showing three phase transitions with temperature (solid to fluid to solid to fluid). The concentrations refer to surface concentration in the case of the grafted strands and solution concentrations in the case of the displacing strands. Surface concentrations are estimated from the expected number of DNA strands of each type, assuming a total of 6500 DNA strands per particle confined to a shell the width of the radius of gyration of the DNA $(11 \mathrm{~nm})$.

\begin{tabular}{|c|c|c|c|}
\hline Particle & Strand name & Base Sequence & Concentration \\
\hline$\overline{\mathrm{red}}$ & $5 \mathrm{~A}$ & $5^{\prime}-\left(\mathrm{T}_{54}\right)$-CA ATG GAG GCT-3' & $150 \mu \mathrm{M}$ \\
\hline red & $5 \mathrm{C}$ & $5^{\prime}-\left(\mathrm{T}_{59}\right)-\mathrm{CAG}$ GTG-3' & $150 \mu \mathrm{M}$ \\
\hline undyed & $5 B$ & $5^{\prime}-\left(\mathrm{T}_{54}\right)-\mathrm{TA}$ TAG CCT CCA-3' & $112.5 \mu \mathrm{M}$ \\
\hline undyed & $5 \mathrm{E}$ & 5'-(T T $\left._{59}\right)$-CAC CTG-3' & $75 \mu \mathrm{M}$ \\
\hline - & $5 D_{1}$ & 5'-CT CCA TTG-3' & $125 \mu \mathrm{M}$ \\
\hline - & $5 D_{2}$ & 5'-AG GCT ATA-3' & $125 \mu \mathrm{M}$ \\
\hline
\end{tabular}

Table 6 Thermodynamic parameters for sequences used for the system with three phase transitions, calculated using the nearest-neighbor model at $250 \mathrm{mM} \mathrm{NaCl}$.

\begin{tabular}{lll}
\hline Duplex name & $\Delta H(\mathrm{kcal} / \mathrm{mol})$ & $\Delta S(\mathrm{cal} / \mathrm{mol} / \mathrm{K})$ \\
\hline $5 A 5 B$ & -53.5 & -145.6 \\
$5 A 5 D_{1}$ & -55.6 & -157.9 \\
$5 B 5 D_{2}$ & -50.4 & -143.8 \\
$5 C 5 E$ & -41.0 & -115.9 \\
\hline
\end{tabular}

We estimate that as many as nine phase transitions can be incorporated into a single system, assuming that crosstalk between the DNA strands can be avoided and that each of the grafted strands is in high enough surface concentration to allow the melting transition to occur at the desired temperature (see Figure 3). The limits are set by the regime in which water is liquid (0$100{ }^{\circ} \mathrm{C}$ ) and by the minimum width of the regime where the solid phase is stable-approximately $10^{\circ} \mathrm{C}$, as discussed in Section 5.2.

\subsection{Compositional transitions}

All of the examples that we have shown thus far involve two species of particles. We now show that the same displacementmediated interactions can be used in systems with multiple species. We demonstrate an experimental system with a controlled transition between binary crystals with different compositions (Figure 7).

This system contains three different particle species (shown as red, green, and blue in the figure). Similarly to the system discussed in Section 6.1, the green species is grafted with two different sequences of DNA that allow it to bind to both the red and the blue species. The sequences are designed such that at low temperature, the blue and green particles are bound while the red particles remain in a fluid state. At high temperature, the red and green particles are bound while the blue particles are in a fluid state. These two regimes are separated by a $10{ }^{\circ} \mathrm{C}$ window in which the entire system is a fluid (Figure 7b).
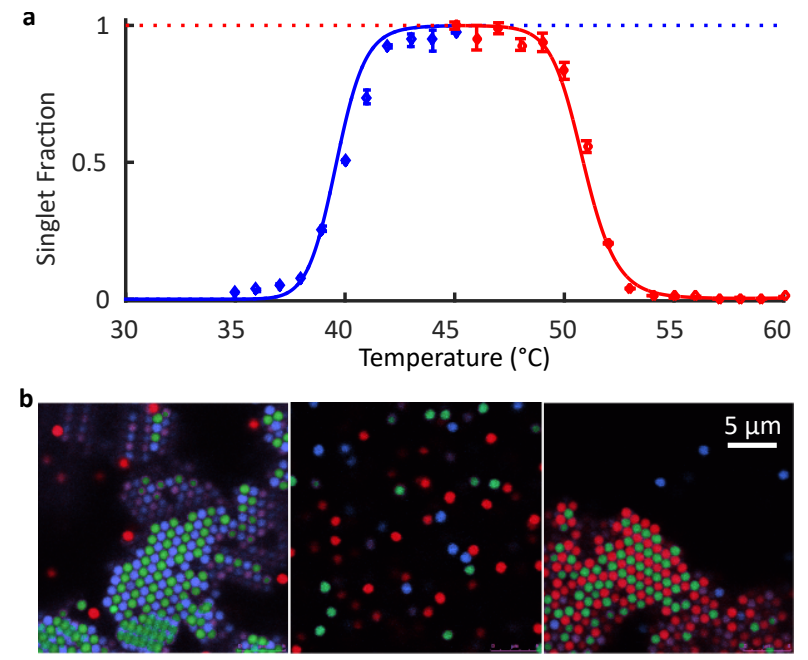

Fig. 7 a) Experimental singlet fraction measurements for blue/green interactions (blue symbols) and red/green interactions (red symbols) with a fit to the model overlaid (lines). b) Confocal fluorescence micrographs showing the transition from a blue/green crystal phase at low temperature to a red/green crystal phase at high temperature through an intermediate fluid phase. The intermediate fluid phase is used to anneal the crystal into the two phases on cooling or heating. The displacing-strand concentration used is $C_{D_{1}}=C_{D_{2}}=62.5 \mu \mathrm{M}$.

Table 7 DNA sequences used for compositional transitions. Concentrations are defined as in Table 5.

\begin{tabular}{|c|c|c|c|}
\hline Particle & Strand name & Base Sequence & Concentration \\
\hline$\overline{\text { red }}$ & $\bar{A}$ & 5'-(T $\left.T_{51}\right)$-CT AAC TGC GGT-3' & $300 \mu \mathrm{M}$ \\
\hline green & B & 5'-(T $\left.\mathrm{T}_{51}\right)$-CT TAC CGC AGT-3' & $150 \mu \mathrm{M}$ \\
\hline green & $\mathrm{E}$ & 5'-(T⿰亻 & $150 \mu \mathrm{M}$ \\
\hline blue & $\mathrm{F}$ & $5^{\prime}-\left(\mathrm{T}_{59}\right)-\mathrm{CAC}$ CTG-3' & $300 \mu \mathrm{M}$ \\
\hline - & $D_{1}$ & 5'-GC AGT TAG-3' & $62.5 \mu \mathrm{M}$ \\
\hline - & $D_{2}$ & 5'-GC GGT AAG-3' & $62.5 \mu \mathrm{M}$ \\
\hline
\end{tabular}

Table 8 Thermodynamic parameters for sequences used for compositional transitions, calculated using the NUPACK software for $500 \mathrm{mM}$ $\mathrm{NaCl}$.

\begin{tabular}{lll}
\hline Duplex name & $\Delta H(\mathrm{kcal} / \mathrm{mol})$ & $\Delta S(\mathrm{cal} / \mathrm{mol} / \mathrm{K})$ \\
\hline$A B$ & -63.6 & -171.5 \\
$A D_{1}$ & -64.6 & -184.8 \\
$B D_{2}$ & -69.6 & -194.1 \\
$E F$ & -49.0 & -138.0 \\
\hline
\end{tabular}

This phase behavior is designed through the combination of a displacement-free interaction between the green and blue particles and a two-displacement interaction between the red and green particles (Tables 7 and 8). The blue and green particles, which are grafted with complementary DNA, bind together at low temperature and unbind above $40^{\circ} \mathrm{C}$. The green particles are grafted with a second strand of DNA that allows them to interact with the red particles through a two-displacing-strand interaction, such that they are dispersed at low temperatures and aggregated at temperatures above $51{ }^{\circ} \mathrm{C}$ (and eventually redisperse at temperatures higher than those reached in the experiment). Therefore, the same system can be driven to assemble into two crystals of different composition. Both the equilibrium structure of the phases and the transitions between these phases are encoded in 
the DNA sequences.

\section{Conclusions and practical considerations}

We have shown, through theory and experiment, that stranddisplacement reactions are a powerful way to alter-in fundamental ways - the temperature dependence of DNA-mediated interactions between colloidal particles. Using a small number of experimental building blocks, one can create broadened melting transitions, temperature-independent coexistence between fluid and solid phases, and inverted transitions where particles bind with increasing strength as temperature increases. A summary of the phase behaviors that can be achieved with no displacing strands, a single strand-displacement reaction, and two strand-displacement reactions is shown in Figure 8. The diversity of the melting curves highlights the versatility of the stranddisplacement schemes. We have also shown that these schemes can be combined with multiple strands of DNA on a single species of particle to realize more complicated phase transitions or with multiple species of particles to realize compositional transitions.

The model that we present provides a theoretical basis for the change in phase behavior resulting from changes in the concentrations of the displacing strands in solution. It also allows us to design the DNA sequences required to realize these systems experimentally.

This strand-displacement toolkit may make it easier to assemble DNA-grafted colloidal particles. For example, broadening the melting transition could allow for easier annealing of structures. This effect is especially important in systems containing multiple species of particles. In such systems the melting temperatures of different pairs of species must be matched so that they can all anneal in the same temperature range. It is much easier to match these temperatures by controlling the concentration of displacing strand in solution than by trying to control the grafted strand concentrations, which requires modifying the synthesis of the particles. Also, the ability to use several species of particles in the same system, where each pair can exhibit phase behavior with multiple transitions, opens the door to systems that can dynamically reconfigure between multiple structures as the temperature is tuned.

Incorporating displacement reactions into a DNA-grafted system is straightforward-so much so, in fact, that we now design all of our DNA-grafted particles so that there are "toeholds" on each strand for displacement reactions to occur, as shown in Figure 1c. By adding toeholds, we always have the choice to use displacement reactions. If we choose to use them, we can simply add the displacing strands to the solution, and we can easily control the shape of the melting transition(s) by varying the concentration of free strands. The displacing strands, being a few bases long, are inexpensive and do not require purification.

If we choose not to include displacement reactions, there is no harm in including the toeholds, so long as we are careful to avoid crosstalk between the toeholds and the binding domains. The toeholds are designed such that the free energy of hybridization between the grafted and displacing strands is the same as that between the grafted strands, as discussed above. Practically, we find that it is easiest to meet this constraint and avoid crosstalk through a few guidelines. First, we avoid sequences with more than two $\mathrm{C}$ or $\mathrm{G}$ bases in a row. These repeats lead to unintended interactions and hairpins, owing to the high stability of C-G bonds. Second, we avoid using any base three or more times in a row. Third, we design our toeholds to be three bases long, so that they bind the displacing strands strongly enough to ensure that displacement occurs on short timescales, but weakly enough so that the displacing strands do not remain bound to the grafted strand through the toehold alone. ${ }^{22,23}$ Further details of our DNA sequence design process are discussed in Section 8.1.

Finally, we note that while the experiments here rely on the method shown by Kim, Manoharan, and Crocker ${ }^{33}$ to graft DNA onto the particles, the control over the temperature dependence afforded by displacement reactions should work for particles with much higher surface coverage, including those demonstrated recently by Pine and collaborators. ${ }^{34-36}$ We also foresee no obstacles to integrating strand-displacement reactions into the emulsion systems with the mobile DNA strands demonstrated by Brujic and coworkers. ${ }^{37,38}$

\section{Methods and materials}

\subsection{Sequence design}

All grafted oligonucleotides are 65 bases long, single stranded, and consist of an inert poly-T spacer and a binding domain on the 3' end. The poly-T spacer sets the range of interaction, and the binding-domain sequence sets the strength and specificity of binding.

To design the base sequence of a binding domain, we generate many random sequences of bases of the appropriate length from the complete set of three-base codons (where codon refers to a unique three-base segment of DNA), each used only once to minimize unintended crosstalk and hairpins. We then use either the nearest-neighbor model ${ }^{18}$ or the online software NUPACK $^{32}$, which uses the nearest-neighbor parameters along with other corrections, to calculate the thermodynamic parameters (enthalpy and entropy contribution to binding energy) of each sequence. We calculate these parameters at salt concentrations matching the conditions of the corresponding experimental measurements. We then filter the sequences such that they meet the specifications for the single-displacement scheme of Section 4 $\left(\Delta H_{A B}=\Delta H_{A D_{1}}\right)$ or the two-displacement scheme of Section 5 $\left(\Delta G_{A B}=\Delta G_{A D_{1}}=\Delta G_{B D_{2}}\right)$. We match these thermodynamic parameters within $5 \%$.

Once all of these requirements are met, we ensure that the melting transitions fall in the desired range using a Matlab program (available at https://github.com/manoharan-lab/DNAcolloid-design). This program calculates the singlet fraction as a function of temperature for any given combination of sequences using the model described in Sections 2, 3, 4.1, and 5.1. We then check for stable secondary structures and unintended crosstalk between non-interacting sequences using NUPACK and Mfold ${ }^{39}$. We reject sequences with secondary structures that are stable above $20{ }^{\circ} \mathrm{C}$. We also reject if unintended crosstalk is predicted to occur more than $1 \%$ as often as the desired structure within the working temperature range for the experiment. 

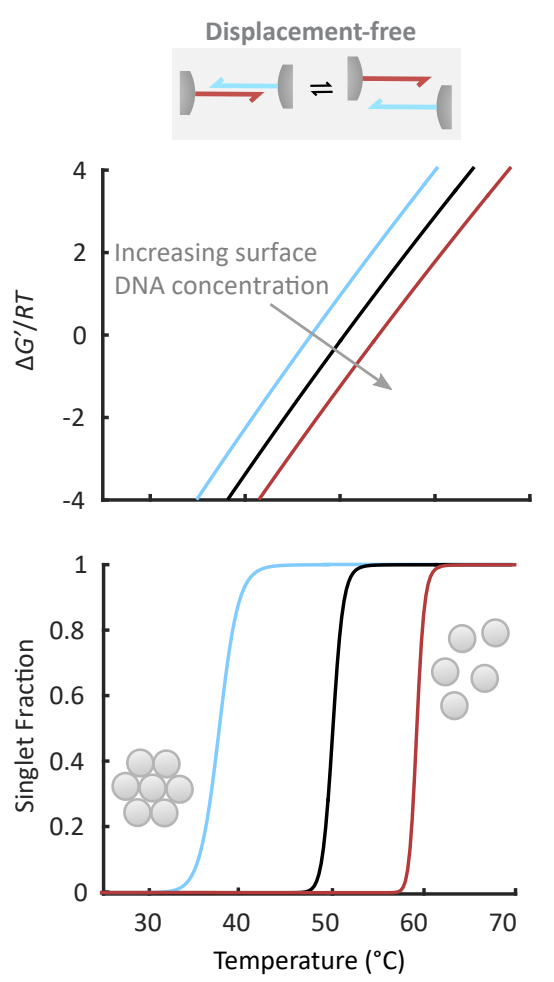

One displacement reaction
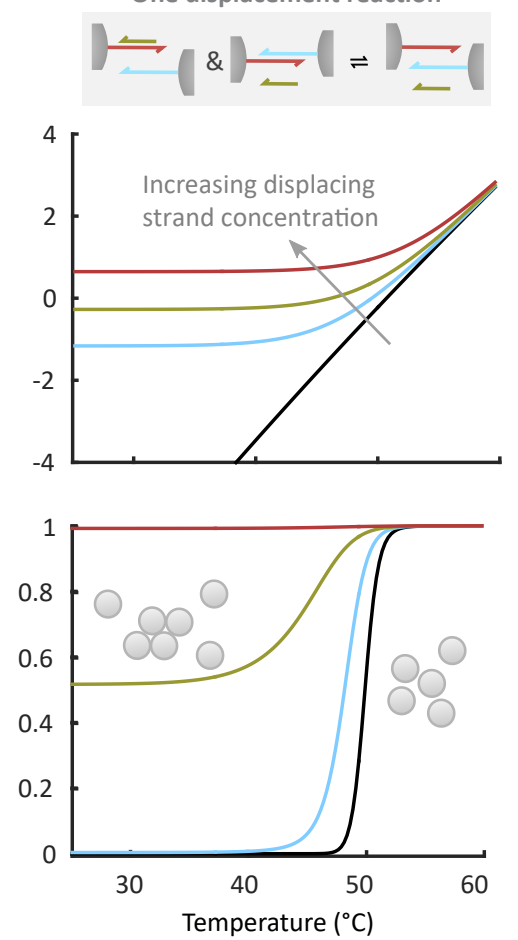
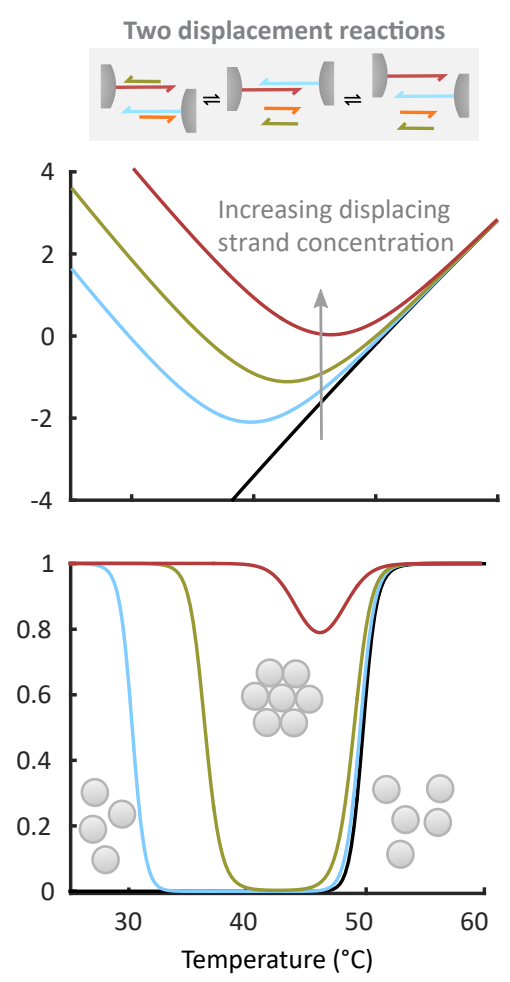

Fig. 8 A summary of the basic phase behaviors that can be achieved with no displacing strands (left), a single strand-displacement reaction (middle), and two strand-displacement reactions (right). Plots of $\Delta G^{\prime}$ (top row) and singlet fraction (bottom row) as a function of temperature calculated using Equations 6, 10, and 13 for $\Delta G^{\prime}$ and Equations 2, 4, 5, and 9 for singlet fraction. The calculations for the black curve on each plot are performed for typical experimental values of the concentrations and thermodynamic parameters: the DNA surface concentration is $C_{A 0}=230 \mu \mathrm{M}$, and the hybridization enthalpy and entropy of the DNA are $\Delta H_{A B}=\Delta H_{A D_{1}}=\Delta H_{B D_{2}}=-63.8 \mathrm{kcal} / \mathrm{mol}$ and $\Delta S_{A B}=\Delta S_{A D_{1}}=\Delta S_{B D_{2}}=-180.3 \mathrm{cal} / \mathrm{mol} / \mathrm{K}$. The surface concentrations used in the displacement-free case are 76.6 (blue) and $690 \mu \mathrm{M}$ (red). The displacing-strand concentrations used in the one-displacement-reaction case are $C_{D_{1}}=76$ (blue), 184 (green), and 460 (red) $\mu \mathrm{M}$. The displacing-strand concentrations used in the two-displacement-reaction case are $C_{D_{1}}=C_{D_{2}}=$ 6.9 (blue), 18.4 (green), and 57.5 (red) $\mu \mathrm{M}$. For one displacement reaction, the concentration-adjusted free energy of the system flattens, leading to a wide coexistence region between fluid and solid. The width of the transition and the degree of coexistence can be tuned with the displacing-strand concentration. For two displacement reactions, the free energy reverses slope at low temperature, leading to a second melting transition. The position of this second, low temperature transition is controlled by the concentration of displacing strands.

We then order single-stranded DNA oligonucleotides, with the sequences we have designed, from Integrated DNA Technologies, Inc. with an amino-C6-modifier added to the 5' end of the surfacegrafted strands. Surface-grafted strands are purified by highperformance liquid chromatography, and short, soluble strands are purified by standard desalting. These purification steps are performed by the supplier.

\subsection{Functionalizing particles}

We functionalize our colloidal particles with DNA by chemically bonding our DNA strands to a triblock copolymer, which we then physically graft to the surface of the particles. Our protocol is modified from that described in reference 33 .

We begin by activating the hydroxyl end groups of a poly(ethylene oxide)-poly(propylene oxide)-poly(ethylene oxide) triblock copolymer (Pluronic F108, BASF) by dissolving $500 \mathrm{mg}$ of Pluronic F108 in $2 \mathrm{~mL}$ of dichloromethane (anhydrous, $\geq 99.8 \%$, Sigma-Aldrich) and $30 \mu \mathrm{L}$ of triethylamine (Sigma-Aldrich). We add $100 \mathrm{mg}$ of $p$-nitrophenyl chloroformate (Sigma-Aldrich), cool the solution to $0^{\circ} \mathrm{C}$ and stir for at least 4 hours to allow the reaction to complete.
After the reaction, we wash the activated Pluronic once in 3\% $\mathrm{v} / \mathrm{v}$ hydrochloric acid (38\% ACS grade, EMD) in ethanol (200 proof, Koptec) and three times in $1 \% \mathrm{v} / \mathrm{v}$ hydrochloric acid in ethanol. After the final wash, we pour off the supernatant and allow the pellet to dry in a vacuum desiccator for at least 7 hours.

We then react the activated Pluronic with 5'-amino-C6modified oligonucleotides (IDT) by first dissolving $15 \mathrm{mg}$ of the activated Pluronic in $1 \mathrm{~mL}$ of $10 \mathrm{mM} \mathrm{pH} 4.0$ citric acid buffer (1.1 mM Anhydrous Citric Acid, EMS 99.5\%, 8.9 mM Sodium Citrate, Spectrum, 99.0-100.5\%). We then combine $15 \mu \mathrm{L}$ of DNA (1 mM in molecular-biology-grade water) with $1 \mu \mathrm{L}$ of $1 \mathrm{M}$ pH 9.5 carbonate buffer (0.85 M Sodium Bicarbonate, EMD 99.7-100.3\%, 0.15 M Sodium Carbonate, EMD $\geq 99.5 \%$ ) and $4 \mu \mathrm{L}$ of the activated Pluronic in citric acid buffer. We allow this solution to react on a room temperature vortexer for at least 4 hours to allow the DNA to bind to the activated end groups of the Pluronic.

Once the reaction has completed, we physically graft the DNAfunctionalized Pluronic to the surface of $1-\mu \mathrm{m}$ polystyrene sulfate particles (Invitrogen). To graft, we first wash the particles in aqueous buffer containing $10 \mathrm{mM}$ Tris and $1 \mathrm{mM}$ EDTA (diluted to 1xTE from Serva TE Buffer (100x), $\mathrm{pH}=8$ ), finishing 
at a total volume fraction of $10 \%$. If the particles do not appear well dispersed, we sonicate until there is no aggregation. After the particles are washed, we combine $340 \mu \mathrm{L}$ of citric acid buffer with $40 \mu \mathrm{L}$ of the DNA-functionalized Pluronic solution and 40 $\mu \mathrm{L}$ of the $10 \%$ particle solution. We then add $4 \mu \mathrm{L}$ of toluene (anhydrous, Sigma) being careful to not strongly agitate the solution through vortexing, sonication, or centrifugation. We allow the solution to mix gently on a room temperature rotator for at least 7 hours to allow the central block of the Pluronic to infiltrate into the swollen matrix of polystyrene. At the end of this time we heat an open container of the solution to $90{ }^{\circ} \mathrm{C}$ in a vented oven for 12 minutes to allow the toluene to evaporate, thus deswelling the particles. Finally we wash the particles at least five times in $1 \mathrm{xTE}$ buffer to remove any excess Pluronic.

We estimate our total DNA density to be 6,500 DNA strands per particle by flow cytometry and melting-curve measurements, and we use this value in all calculations presented. We are easily able to incorporate hydrophobic dyes, such as Coumarin 545 (Exciton) or BODIPY 558/568 (Life Technologies), or a mixture of the two, into the polystyrene core by dissolving them in the toluene used for swelling.

\subsection{Sample preparation}

Functionalized colloidal particles are stored separately in a $4{ }^{\circ} \mathrm{C}$ refrigerator at $1 \%$ in 1xTE (Tris-EDTA ph 8.0 ) buffer. When we need to prepare a sample, we mix the particles with displacing strands at appropriate concentrations in a 1xTE solution with a final concentration of $250 \mathrm{mM} \mathrm{NaCl}$. Exceptions are the singletfraction measurements for the single-displacement-reaction case, where the samples were prepared with $50 \mathrm{mM} \mathrm{NaCl}$ and $10 \mathrm{mM}$ $\mathrm{MgCl}_{2}$, and the singlet-fraction measurements for the system with three species of particles, where the samples were prepared at $500 \mathrm{mM} \mathrm{NaCl}$. This solution is then loaded into a sample chamber prepared as described in the following three subsections.

\subsection{Performing singlet-fraction experiments}

Melting-curve measurements are performed in 2D sample chambers to allow for comparison to the theoretical singlet-fraction equations discussed in Section 2.

Samples are made of a 1:1 mixture of the two particles species at a total volume fraction of $4 \%$. This solution is sealed in a $2 \mathrm{D}$ sample chamber made from two coverslips (No. 1; VWR) that we plasma-clean for approximately 1 minute to prevent nonspecific binding of the DNA-grafted microspheres to the glass surfaces. 1.8- $\mu \mathrm{m}$-diameter silica microspheres are deposited to provide the correct spacing between the coverslips for $2 \mathrm{D}$ confinement. Once the sample is added, the coverslips are bonded together and sealed by UV-curable optical adhesive (Norland 63).

The sample is imaged on an inverted optical microscope (Nikon TE2000-E) under $100 \times$ magnification. The sample temperature is controlled by a resistive heater (Bioscience Tools) wrapped around the objective, which is in contact with the sample through immersion oil. This heater is driven by a low-noise temperature controller (Bioscience Tools). The sample is also heated using a thermoelectric cooler (TE Technology, Inc.) bonded directly to the sample by silicone vacuum grease. The thermoelectric cooler is driven by a separate high-performance digital temperature controller designed to drive thermoelectric cooler elements (Thorlabs). The sample is equilibrated at each temperature point for about $15 \mathrm{~min}$ before data is acquired. Each data point represents the average of three frames at a given temperature. Each frame is approximately $10^{4} \mu \mathrm{m}^{2}$ and contains about 1000 particles.

The singlet fraction is determined using traditional image analysis routines. ${ }^{40}$ We account for the systematic bias arising from the presence of particles that are in close proximity but unbound by comparing our measured singlet fraction to a simple Monte Carlo simulation of hard disks at the same concentration as our experiments.

\subsection{Performing phase behavior experiments}

Phase behavior experiments such as those used to produce Figure $6 \mathrm{~b}$ are performed on an inverted optical microscope (Nikon TE2000-E) under $100 \times$ magnification.

Samples are prepared by making a 1:1 mixture of particles at a total volume fraction of $0.5 \%$. The sample is sealed between two plasma-cleaned coverslips using silicone vacuum grease (Dow Corning). The space between the coverslips in the resulting sample chambers is about $30 \mu \mathrm{m}$. Thus the particles can move about in a $3 \mathrm{D}$ volume.

The sample is heated as described in Section 8.4.

The phase of the sample is determined by visual inspection of micrographs and videos of the sample. In cases where the fraction of unbound particles does not clearly fall into one of the three categories described in Section 6.1, the number of unbound particles in a single field of view is manually counted and compared to the number of unbound particles for the same sample far above the melting temperature.

\subsection{Performing confocal experiments}

Confocal experiments are performed on a Leica SP5 confocal microscope. For general confocal experiments (for example, Figures 2 and 6c), samples are prepared as described in Section 8.5.

For confocal crystallization experiments (for example, Figure 7), samples are prepared by making a 1:1 mixture of particles at a total volume fraction of $5 \%$ and density matching the particles to the solvent by adding $6 \% \mathrm{w} / \mathrm{w}$ sucrose to the buffer. The sample is sealed and heated as in the general experiments.

All two and three-color images from confocal fluorescence microscopy experiments are produced from two-color images, which are measured when two detectors simultaneously collect emitted photons in different wavelength bands. The signals from these detectors become red $\left(r^{\prime}\right)$ and blue $\left(b^{\prime}\right)$ channels. Red $(r)$, green $(g)$, and blue $(b)$ channels of the three-color images in Figure 7 are computed according to:

$$
\begin{gathered}
r=r^{\prime}-\sqrt{r^{\prime} b^{\prime}} \\
g=\sqrt{r^{\prime} b^{\prime}} \\
b=b^{\prime}-\sqrt{r^{\prime} b^{\prime}} .
\end{gathered}
$$




\subsection{Fitting the models to experimental data}

In Figures 4, 5, 6, and 7 we fit the model from Sections 2, 3, 4.1, 5.1, and 6.1 to experimentally measured singlet-fraction curves using non-linear least squares fitting.

For the single-displacement case in Figure 4, we use two different methods to perform the fits. In the first method (black and gray lines), we fit the simplest version of the model, defined by Equations 2, 4, 9, and 10, where $\Delta H_{A B}, \Delta H_{A D_{1}}, \Delta S_{A B}$, and $\Delta S_{A D_{1}}$ are the fit parameters. This simplified model is simultaneously fit to all of the data for the five different displacing-strand concentrations. The fitted values returned are $\Delta H_{A B}=-57.28 \mathrm{kcal} / \mathrm{mol}$, $\Delta S_{A B}=-165.06 \mathrm{cal} /(\mathrm{mol} \mathrm{K}), \Delta H_{A D_{1}}=-58.05 \mathrm{kcal} / \mathrm{mol}$, and $\Delta S_{A D_{1}}=-159.66 \mathrm{cal} /(\mathrm{mol} \mathrm{K})$.

In the second method (shown in red), we fit the full model defined by Equation 3, including the interparticle repulsive discussed in reference 26 . The fit parameters are the same four thermodynamic parameters as above. This fitting method is described in reference 1 . The fitted values returned are $\Delta H_{A B}=$ $-55.46 \mathrm{kcal} / \mathrm{mol}, \Delta S_{A B}=-159.07 \mathrm{cal} /(\mathrm{mol} \mathrm{K}), \Delta H_{A D_{1}}=-55.21$ $\mathrm{kcal} / \mathrm{mol}$, and $\Delta S_{A D_{1}}=-150.51 \mathrm{cal} /(\mathrm{mol} \mathrm{K})$.

For the two-displacement case in Figures 5 and 7, we fit only the simplified model based on Equations 2, 4, 9, and 13. Here we fit each curve separately. First we fit the measurements for the system with no displacing strands for $\Delta H_{A B}$, holding $\Delta S_{A B}$ fixed at the value predicted using NUPACK (Table 4). For each of the remaining curves, we hold $\Delta H_{A B}$ and $\Delta S_{A B}$ constant and use a single fit parameter $f$, which serves as a common multiplicative factor to the enthalpic changes on hybridization of the two displacing strands: $f \Delta H_{A D_{1}}$ and $f \Delta H_{B D_{2}}$. We fix $\Delta S_{A D_{1}}$ and $\Delta S_{B D_{2}}$ to the values predicted from the nearest-neighbor model using NUPACK. The final fitted values are: $\Delta H_{A B}=-64.40 \mathrm{kcal} / \mathrm{mol}, f=1.016$ (for $C_{D}=31.25 \mu \mathrm{M}$ ), $f=1.024$ (for $C_{D}=62.5 \mu \mathrm{M}$ ), $f=1.028$ (for $C_{D}=125 \mu \mathrm{M}$ ), and $f=1.033$ (for $C_{D}=250 \mu \mathrm{M}$ ).

For the three-species system shown in Section 6.2 and Figure 7, we perform two separate fits for the interactions between the two different pairs of particles. For the displacement-free interaction between the blue and green particles, we fit the model given in Sections 2 and 3 for $\Delta H_{A B}$, holding $\Delta S_{A B}$ fixed at the value predicted by NUPACK (Table 8 ). This fit returns $\Delta H_{A B}=48.59$ $\mathrm{kcal} / \mathrm{mol}$.

For the two-displacement-strand reaction between the red and green particles, we simultaneous fit for $\Delta H_{A B}$ and for a common multiplicative factor $f$ to the enthalpic changes on hybridization of the two displacing strands: $f \Delta H_{A D_{1}}$ and $f \Delta H_{B D_{2}}$. We hold the entropic changes on hybridization fixed at the value predicted by NUPACK (Table 8). The final fitted values are $\Delta H_{A B}=-64.2314$ $\mathrm{kcal} / \mathrm{mol}$ and $f=1.0147$.

In the system with three transitions (Section 6.1), we again consider the simplified model, now given by Equation 15 . Although our measurements are now performed in 3D, we calculate the singlet fraction using the 2D model given by Equation 4. For simplicity, we keep the particle coordination number and areal particle concentration the same as for the 2D measurements. The model depends on $\Delta H_{A B}, \Delta H_{A D_{1}}, \Delta H_{B D_{2}}, \Delta H_{C E}$, and the corresponding entropic changes on hybridization for those four du- plexes. We set all of these quantities to the values predicted by the nearest-neighbor model (Table 6) and then fit for a multiplicative factor for each of them. To simplify the fit, we constrain the enthalpic and entropic changes to vary together for each set of sequences, and we further constrain the enthalpic and entropic changes for both of the displacement reactions to vary by a common factor. The result of these constraints is a set of three fitting parameters, $f_{1}, f_{2}$, and $f_{3}$, where our thermodynamic changes on hybridization become $f_{1} \Delta H_{A B}, f_{1} \Delta S_{A B}$, $f_{2} \Delta H_{A D_{1}}, f_{2} \Delta S_{A D_{1}}, f_{2} \Delta H_{B D_{2}}, f_{2} \Delta S_{B D_{2}}, f_{3} \Delta H_{C E}, f_{3} \Delta S_{C E}$.

We fit these three factors separately by first considering the phase-behavior data for the case with no displacing strands. We manually vary $f_{1}$ until the melting transition for the model occurs in the location indicated by the experimental data (around $52.5^{\circ} \mathrm{C}$ ). We then consider the case with high concentration of displacing strands $\left(C_{D 1}=C_{D 2}=225 \mu \mathrm{M}\right)$, where we manually vary $f_{3}$ so that the melting transition of the model matches the observed phase transition at $30^{\circ} \mathrm{C}$. Finally, we hold $f_{1}$ and $f_{3}$ constant at the values found using this procedure, and we manually vary $f_{2}$ until the model for the singlet fraction simultaneously fits the observed transitions for the remaining 5 experimental data sets: $C_{D 1}=C_{D 2}=75,100,125,150,175 \mu \mathrm{M}$. The fitted values found for these multiplicative factors are $f_{1}=1.04, f_{2}=1.11$, and $f_{3}=1.07$.

\section{Acknowledgments}

We thank Michael Brenner for helpful discussions. E.W.G. acknowledges support from the Department of Defense through the National Defense Science \& Engineering Graduate Fellowship (NDSEG) Program. V.N.M. acknowledges support from an Alfred P. Sloan research fellowship. This work was funded by the National Science Foundation through grant no. DMR-1435964 and by the Harvard MRSEC through grant no. DMR-1420570.

\section{Conflict of interest}

There are no conflicts to declare.

\section{References}

1 W. B. Rogers and V. N. Manoharan, Science, 2015, 347, 639642.

2 C. A. Mirkin, R. L. Letsinger, R. C. Mucic and J. J. Storhoff, Nature, 1996, 382, 607-609.

3 A. P. Alivisatos, K. P. Johnsson, X. Peng, T. E. Wilson, C. J. Loweth, M. P. Bruchez and P. G. Schultz, Nature, 1996, 382, 609-611.

4 P. L. Biancaniello, A. J. Kim and J. C. Crocker, Physical Review Letters, 2005, 94, 058302.

5 W. B. Rogers, W. M. Shih and V. N. Manoharan, Nature Reviews Materials, 2016, 1, 16008.

6 A. J. Kim, P. L. Biancaniello and J. C. Crocker, Langmuir, 2006, 22, 1991-2001.

7 S. Y. Park, A. K. Lytton-Jean, B. Lee, S. Weigand, G. C. Schatz and C. A. Mirkin, Nature, 2008, 451, 553-556.

8 D. Nykypanchuk, M. M. Maye, D. van der Lelie and O. Gang, Nature, 2008, 451, 549-552. 
9 J. T. McGinley, I. Jenkins, T. Sinno and J. C. Crocker, Soft Matter, 2013, 9, 9119-9128.

10 E. Ducrot, M. He, G.-R. Yi and D. J. Pine, Nature Materials, 2017, 16, 652-657.

11 Y. Wang, I. C. Jenkins, J. T. McGinley, T. Sinno and J. C. Crocker, Nature Communications, 2017, 8, 14173.

12 R. J. Macfarlane, B. Lee, M. R. Jones, N. Harris, G. C. Schatz and C. A. Mirkin, Science, 2011, 334, 204-208.

13 Y. Zhang, S. Pal, B. Srinivasan, T. Vo, S. Kumar and O. Gang, Nature Materials, 2015, 14, 840-847.

14 M. M. Maye, M. T. Kumara, D. Nykypanchuk, W. B. Sherman and O. Gang, Nature Nanotechnology, 2010, 5, 116-120.

15 R. Dreyfus, M. E. Leunissen, R. Sha, A. V. Tkachenko, N. C. Seeman, D. J. Pine and P. M. Chaikin, Physical Review Letters, 2009, 102, 048301.

16 Q. Xu, L. Feng, R. Sha, N. C. Seeman and P. M. Chaikin, Physical Review Letters, 2011, 106, 228102.

17 K.-T. Wu, L. Feng, R. Sha, R. Dreyfus, A. Y. Grosberg, N. C. Seeman and P. M. Chaikin, Physical Review E, 2013, 88, 022304.

18 J. SantaLucia and D. Hicks, Annual Review of Biophysics and Biomolecular Structure, 2004, 33, 415-440.

19 J. SantaLucia and D. H. Turner, Biopolymers, 1997, 44, 309319.

20 B. Yurke, A. J. Turberfield, A. P. Mills, F. C. Simmel and J. L. Neumann, Nature, 2000, 406, 605-608.

21 D. Y. Zhang and G. Seelig, Nature Chemistry, 2011, 3, 103113.

22 D. Y. Zhang and E. Winfree, Journal of the American Chemical Society, 2009, 131, 17303-17314.

23 N. Srinivas, T. E. Ouldridge, P. Šulc, J. M. Schaeffer, B. Yurke, A. A. Louis, J. P. K. Doye and E. Winfree, Nucleic Acids Research, 2013, 41, 10641-10658.

24 C. K. Tison and V. T. Milam, Langmuir, 2007, 23, 9728-9736.

25 B. A. Baker, G. Mahmoudabadi and V. T. Milam, Soft Matter, 2013, 9, 11160-11172.

26 W. B. Rogers and J. C. Crocker, Proceedings of the National Academy of Sciences, 2011, 108, 15687-15692.

27 B. M. Mognetti, P. Varilly, S. Angioletti-Uberti, F. J. MartinezVeracoechea, J. Dobnikar, M. E. Leunissen and D. Frenkel, Proceedings of the National Academy of Sciences, 2012, 109, E378-E379.

28 W. B. Rogers and J. C. Crocker, Proceedings of the National Academy of Sciences, 2012, 109, E380-E380.

29 D. Y. Zhang, S. X. Chen and P. Yin, Nature Chemistry, 2012, 4, 208-214.

30 R. Dreyfus, M. E. Leunissen, R. Sha, A. Tkachenko, N. C. Seeman, D. J. Pine and P. M. Chaikin, Physical Review E, 2010, 81, 041404.

31 W. B. Rogers, T. Sinno and J. C. Crocker, Soft Matter, 2013, 9, 6412-6417.

32 J. N. Zadeh, C. D. Steenberg, J. S. Bois, B. R. Wolfe, M. B. Pierce, A. R. Khan, R. M. Dirks and N. A. Pierce, Journal of Computational Chemistry, 2011, 32, 170-173.
33 A. J. Kim, V. N. Manoharan and J. C. Crocker, Journal of the American Chemical Society, 2005, 127, 1592-1593.

34 Y. Wang, Y. Wang, X. Zheng, E. Ducrot, J. S. Yodh, M. Weck and D. J. Pine, Nature Communications, 2015, 6, 7253.

35 J. S. Oh, Y. Wang, D. J. Pine and G.-R. Yi, Chemistry of Materials, 2015, 27, 8337-8344.

36 Y. Wang, Y. Wang, X. Zheng, E. Ducrot, M.-G. Lee, G.-R. Yi, M. Weck and D. J. Pine, Journal of the American Chemical Society, 2015, 137, 10760-10766.

37 L. Feng, L.-L. Pontani, R. Dreyfus, P. Chaikin and J. Brujic, Soft Matter, 2013, 9, 9816-9823.

38 Y. Zhang, A. McMullen, L.-L. Pontani, X. He, R. Sha, N. C. Seeman, J. Brujic and P. M. Chaikin, Nature Communications, 2017, 8, 21.

39 M. Zuker, Nucleic Acids Research, 2003, 31, 3406-3415.

40 J. C. Crocker and D. G. Grier, Journal of Colloid and Interface Science, 1996, 179, 298-310. 
A method for creating broadened, inverted, or multiple phase transitions between colloidal particles grafted with DNA. 
Increasing temperature

$5 \mu \mathrm{m}$

Coft MAOttor

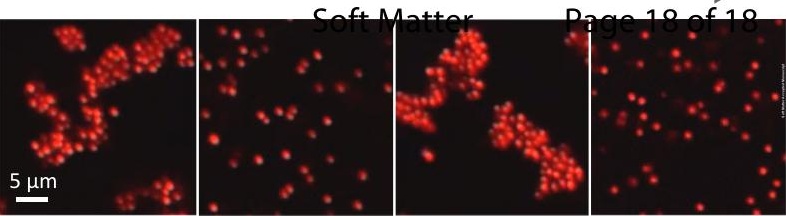

